\title{
Miniature spectral imaging device for wide-field quantitative functional imaging of the morphological landscape of breast tumor margins
}

Brandon S. Nichols

Antonio Llopis

Gregory M. Palmer

Samuel S. McCachren, III

Ozlem Senlik

David Miller

Martin A. Brooke

Nan M. Jokerst

Joseph Geradts

Rachel Greenup

Nimmi Ramanujam 


\title{
Miniature spectral imaging device for wide-field quantitative functional imaging of the morphological landscape of breast tumor margins
}

\author{
Brandon S. Nichols, ${ }^{a}$ Antonio Llopis, ${ }^{b}$ Gregory M. Palmer, ${ }^{c}$ Samuel S. McCachren III, ${ }^{\text {a }}$ Ozlem Senlik, ${ }^{b}$ \\ David Miller, ${ }^{b}$ Martin A. Brooke, ${ }^{b}$ Nan M. Jokerst, ${ }^{b}$ Joseph Geradts, ${ }^{d}$ Rachel Greenup, ${ }^{e}$ and Nimmi Ramanujam ${ }^{a, *}$ \\ ${ }^{a}$ Duke University, Pratt School of Engineering, Department of Biomedical Engineering, Durham, North Carolina, United States \\ ${ }^{\mathrm{b}}$ Duke University, Pratt School of Engineering, Department of Electrical Engineering, Durham, North Carolina, United States \\ 'Duke University Medical Center, Department of Radiation Oncology, Medicine Circle, Durham, North Carolina, United States \\ ${ }^{\mathrm{d} D u k e}$ University Medical Center, Department of Pathology, Durham, North Carolina, United States \\ eDuke University Medical Center, Department of Surgery, Durham, North Carolina, United States
}

\begin{abstract}
We have developed a portable, breast margin assessment probe leveraging diffuse optical spectroscopy to quantify the morphological landscape of breast tumor margins during breast conserving surgery. The approach presented here leverages a custom-made 16-channel annular photodiode imaging array (arranged in a $4 \times 4$ grid), a raster-scanning imaging platform with precision pressure control, and compressive sensing with an optimized set of eight wavelengths in the visible spectral range. A scalable Monte-Carlo-based inverse model is used to generate optical property $\left[\mu_{\mathrm{s}}^{\prime}(\lambda)\right.$ and $\left.\mu_{\mathrm{a}}(\lambda)\right]$ measures for each of the 16 simultaneously captured diffuse reflectance spectra. Subpixel sampling $(0.75 \mathrm{~mm})$ is achieved through incremental $x, y$ raster scanning of the imaging probe, providing detailed optical parameter maps of breast margins over a $2 \times 2 \mathrm{~cm}^{2}$ area in $\sim 9$ min. The morphological landscape of a tumor margin is characterized using optical surrogates for the fat to fibroglandular content ratio, which has demonstrated diagnostic utility in delineating tissue subtypes in the breast. (C) 2017 Society of Photo-Optical Instrumentation Engineers (SPIE) [DOI: 10.1117/1.JBO.22.2.026007]
\end{abstract}

Keywords: optics; photonics; diffuse optical spectroscopy; medical instrumentation; clinical applications; light propagation in tissue. Paper 160658R received Sep. 23, 2016; accepted for publication Jan. 26, 2017; published online Feb. 22, 2017.

\section{Introduction}

Women with stage I or II breast cancer are eligible for a surgical procedure known as breast conserving surgery (BCS). BCS involves removing the tumor and a minimally thin peripheral layer or "margin" of disease-free tissue surrounding the tumor; this procedure is more clinically referred to as a partial mastectomy or lumpectomy. BCS has been shown to be as effective as a total mastectomy when combined with radiation therapy and is therefore the more frequent surgical choice for patients with early stage breast cancer. Among women, breast cancer has the highest incidence rate worldwide and remains the leading cause of cancer-related deaths in developed countries. ${ }^{1}$ It is estimated that $38 \%$ to $65 \%$ of patients eligible for BCS will choose to have the procedure, ${ }^{2} 25 \%$ of whom will be advised to have a second surgery due to the finding of malignant cells at or near the margin of the breast specimen at final pathology. ${ }^{3,4}$ The inability to detect malignant cells within the vicinity of the margin edge during surgery poses a significant unmet clinical need; the surgeon cannot definitively resolve the possibility of incomplete disease excision during the initial surgery, which may require the patient to return for an additional surgery due to an increased risk in local recurrence of disease. The current margin assessment gold standard relies on postoperative histopathology, wherein 4 to $5 \mu \mathrm{m}$ slices are taken from larger 3 to $4 \mathrm{~mm}$ sections of the tumor margin. Although an assessment of the tumor margin status can be made using cytologic or histopathological techniques during surgery, only specialized facilities have the resources to implement them intraoperatively. ${ }^{5,6}$

The primary challenge with intraoperative breast margin assessment is the ability to resolve focal residual disease $\left(0.02-10+\mathrm{cm}^{2}\right)$ within a relatively large background area (10 to $100 \mathrm{~cm}^{2}$ ) of mostly benign tissue. This is further complicated by a need to thoroughly survey the entire tissue surface area in a time frame that is practical for an intraoperative setting. Although there is no standardized procedure time, the typical lumpectomy procedure usually starts with sentinel node mapping to determine if the malignancy has spread to the lymph nodes. The basis for this is that the lumpectomy procedure can be performed while the sentinel node is sent to pathology for frozen section analysis to determine if the patient needs a complete axillary node dissection. The surgeon will typically remove the lumpectomy within this 20 to 40 min window, suggesting that a favorable intraoperative device would assess the margin within only a fraction of this time, allowing the surgeon to perform additional tasks, such as preparing to close the patient or taking additional tissue shaves if deemed necessary. The sampling resolution of pathology is subject to institutional protocol, most commonly resulting in 3- to 5-mm-thick specimen cross sections wherein $5 \mu \mathrm{m}$ shaves are removed from each thick section and evaluated microscopically. These coarse cross sections represent a practical sampling interval that allows pathologists to 
survey the entire margin surface; however, regions of disease less than $3 \mathrm{~mm}$ in width may be missed.

Given these challenges, many research groups, especially those in biophotonics, regard an optical tool as a pragmatic solution. The current state of the art in photonics technology provides an unequivocal advantage to any histologic or cytologic technique; quantitation of measured optical signals can obviate the need for highly trained personnel by providing a rapid computational prediction on the likelihood of residual disease. Stated differently, the same morphological features used in histopathology can be detected and distinguished on a macroscale, thereby eliminating the aforementioned sampling limitations. The degrees of freedom allow one to trade resolution and coverage area for speed and accuracy, meaning that one could "zoom in" on trouble spots yet reasonably expect to survey the entire tissue surface area in minutes. Among the latest investigational optical imaging technologies, most use low-power, nonionizing radiation from the visible and near-infrared (VIS-NIR) portions of the electromagnetic spectrum. Within this wavelength range, tissue constituents can be determined and quantified through spectral analysis, providing information about the morphological state of tissue as deep as $2 \mathrm{~mm}$ noninvasively.

Our group has investigated several iterations of a multiplexed single point quantitative diffuse reflectance spectroscopy probe that has been extensively validated in multiple tissue-simulating phantom and clinical studies. $^{7-9}$ The current clinical workhorse utilized by our group consists of a quantitative diffuse reflectance imaging probe containing 49 independent full-spectrum $(\lambda=[400: 1: 600] \mathrm{nm})$ fiber-optic channels arranged in a $7 \times 7$ array with a $6-\mathrm{mm}$ pixel pitch $(49 \mathrm{ch})$. The $49 \mathrm{ch}$ device evolved from a similar 8-channel $(8 \mathrm{ch})$ device described in Refs. 8 and 9. The diffuse reflectance spectrum from each channel is analyzed with a feature extraction algorithm based on a fast, scalable Monte-Carlo model developed by Palmer and Ramanujam ${ }^{7}$ to quantitatively determine the absorption spectrum $\left[\mu_{\mathrm{a}}(\lambda)\right]$ and reduce the scattering spectrum $\left[\mu_{\mathrm{s}}^{\prime}(\lambda)\right]$. The technology detects varying amounts of malignancy in the presence of benign tissue by quantifying the margin landscape as a cumulative distribution function (CDF) of the ratio of $\beta$-carotene concentration (absorber) and the wavelength averaged tissue scattering $\left([\beta\right.$-carotene $\left.] /\left\langle\mu_{\mathrm{s}}^{\prime}\right\rangle_{\lambda}\right)$. We have established through histopathological validation that the $[\beta$-carotene $] /\left\langle\mu_{\mathrm{s}}^{\prime}\right\rangle_{\lambda}$ reports on the relative amount of adipose to collagen, glands, and fibrous content; decreased ratios are strongly associated with the presence of residual disease. ${ }^{8}$

The cost and complexity of the fiber-based quantitative diffuse reflectance imaging (QDRI) systems suggested clinical translation to be unlikely, particularly in the construction of a fiber probe that can be scaled to survey areas as large as $100 \mathrm{~cm}^{2}$. The primary goal of our most recent effort centers on transitioning from a research-grade device toward a rapid, lowcost, portable, and easy to manufacture tool that performs comparably to our first generation QDRI system as well as other tools developed for intraoperative margin assessment.

We have previously demonstrated a proof-of-concept probe design representing a pragmatic strategy; an array of individual Si photodetectors (PDs) with centralized illumination apertures was used in lieu of delicate fiber-optic bundles. ${ }^{10}$ Using tissuesimulating liquid phantoms, we demonstrated equivalent optical quantitation accuracy to the fiber-based systems. The primary limitation of this initial prototype stemmed from repurposing the commercial photodiodes; producing the illumination apertures involved a drilling process that proved to be unreliable from a manufacturing standpoint due to the brittleness of the encapsulated Si-based photodiodes. Furthermore, we found that pixel pitch was limited to the physical size of available photodiodes and that high SNR $(>35 \mathrm{~dB})$ could not be obtained due to silicon absorption losses that occurred as light passed through the illumination apertures drilled into the thick $(>2 \mathrm{~mm})$ commercial photodiodes. In this paper, we demonstrate an innovative technological approach to address these challenges and establish a pragmatic, commercially viable solution to this important unmet clinical need. Specifically detailed is the performance and clinical exemplification of a portable breast margin assessment probe (BMAP) that is both cost-effective and commercially scalable. The BMAP provides submillimeter $(0.75 \mathrm{~mm})$ wide-field $256 \mathrm{~cm}^{2}$ surveillance of the morphological composition of breast tumor margins by virtue of a rasterized series of pressure-regulated multispectral images processed into highresolution optical parameter maps. Through multiple probe placements, this strategy enables full margin surveillance $(\mathrm{A}=$ $256 \mathrm{~cm}^{2}$ ) at a resolution of $0.75 \mathrm{~mm}$ in fewer than $10 \mathrm{~min}$ and is reproducible within $1 \%$.

\section{Methods}

\subsection{Breast Margin Assessment Probe Instrumentation}

The BMAP system leverages a custom 16-channel wide-field $\left(\mathrm{FOV}=256 \mathrm{~cm}^{2}\right)$ silicon-based photodiode array (PDA), a custom 16-channel transimpedance integrating amplifier, a xenon light source containing eight 10-nm bandpass optical channels, and a custom computer numeric controlled (CNC) imaging platform with adaptive pressure control. Compressive sensing is achieved through optimized bandpass filter center wavelengths (470, 480, 490, 500, 510, 560, 580, and $600 \mathrm{~nm})$ informed by a genetic algorithm analysis of previous clinical data. ${ }^{11}$ The lamp, imaging platform, and amplifier system are daisy chained to a universal serial bus (USB) hub within the amplifier box, allowing full system control via a laptop and a single USB connection. The control software was developed using the LabVIEW ${ }^{\mathrm{TM}} \mathrm{G}$ language; an object oriented framework was chosen to maintain scalability and incorporate a hardware abstraction layer. The system design is described in detail in the following sections and is diagrammed in Fig. 1.

\subsection{Photodiode Array Design}

In contrast to traditional fiber-optic probes, which require careful weaving and placement of glass fibers, the PDAs are constructed using standard silicon semiconductor fabrication techniques. The feature sizes of the individual photodiodes do not require highly specialized equipment and are achieved using photolithography. Moreover, changes in photodiode density, size, and pitch, are easily achieved with additional low-cost photomasks, affording a simple means to scalability. The specific details of the PDA fabrication process flow, photodiode responsivity, SNR, and cross talk considerations are detailed in Ref. 12. Briefly, the imaging array consists of 16 annular photodiodes formed on an $n$-type silicon epitaxial substrate and is arranged in a $4 \times 4$ array with a $4.5 \mathrm{~mm}$ center-to-center pixel pitch, resulting in a $256 \mathrm{~cm}^{2}$ field of view (FOV). Each of the annular photodiodes consists of a circular active area $(d=$ $2.46 \mathrm{~mm})$ with an etched central aperture $(d=0.75 \mathrm{~mm})$. 


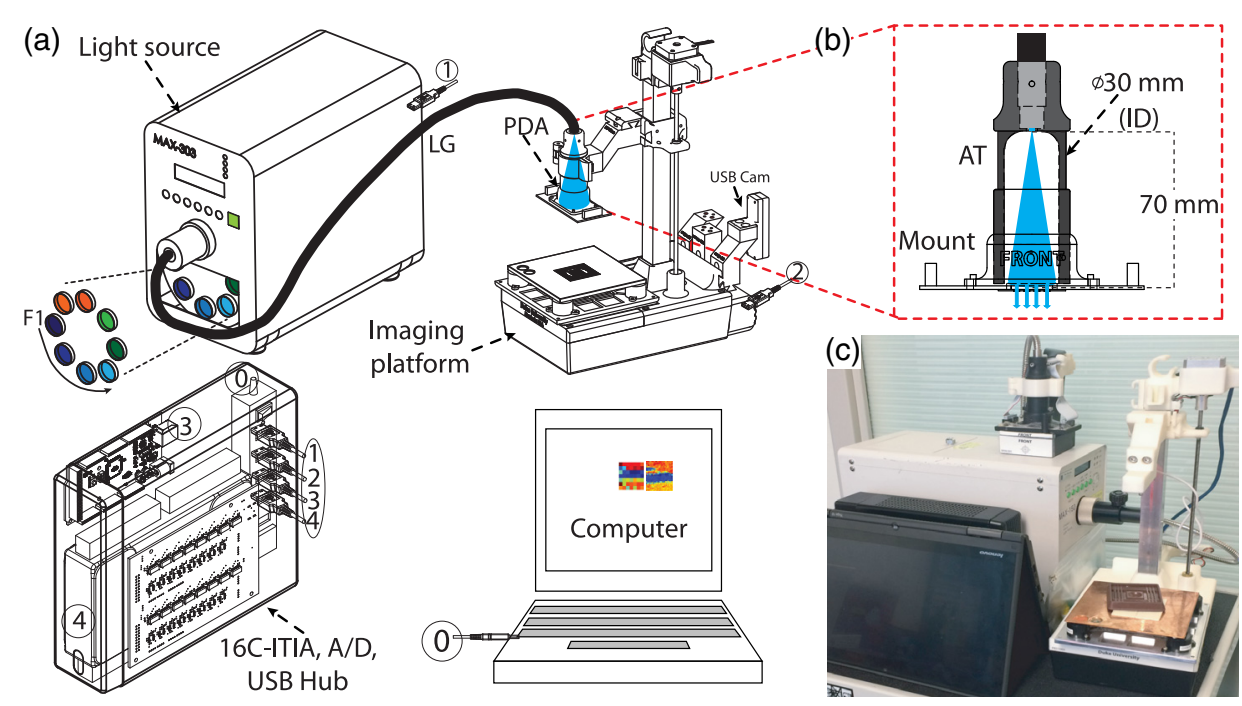

Fig. 1 Diagram of the BMAP system: (a) The system components in clockwise order: a xenon light source with an 8-slot filter wheel containing narrow-band filters $(B P=10 \mathrm{~nm}) \quad(\lambda=[470(\mathrm{~F} 1)$ $480490500510560580600] \mathrm{nm}$ ). Light is delivered to the backside of a PDA using a LG. The LG is coupled to an absorbing free-space tube expanded in (b), delivering low-angle light through the PD apertures. The PDA is clamped to a custom-made imaging platform enabling raster-scanning and controlled probe placement. The light source, imaging platform, and PDA are connected to an amplifier box containing an integrating DAQ and a USB hub to minimize the cable burden, resulting in a single USB connection to a laptop computer. A digital photograph of the system is shown in (c).

The patterned silicon wafer is diced into a rectangle and is epoxied and wire-bonded to a gold plated printed circuit board for structural support and traces for photocurrent readout. An example board-mounted PDA with respective geometry is shown in Fig. 2(a).
On average, the annular photodiodes were observed to have visible light responsivity greater than that of commonly available commercial silicon photodiodes. The measured photodiode responsivity ranged from 0.13 to $0.3 \mathrm{~A} / \mathrm{W}$ for $\lambda=400$ to $600 \mathrm{~nm}$. The SNR, defined as $20 \cdot \log _{10}\left(I_{\text {avg }} \div \sigma_{I}^{2}\right)$, was shown
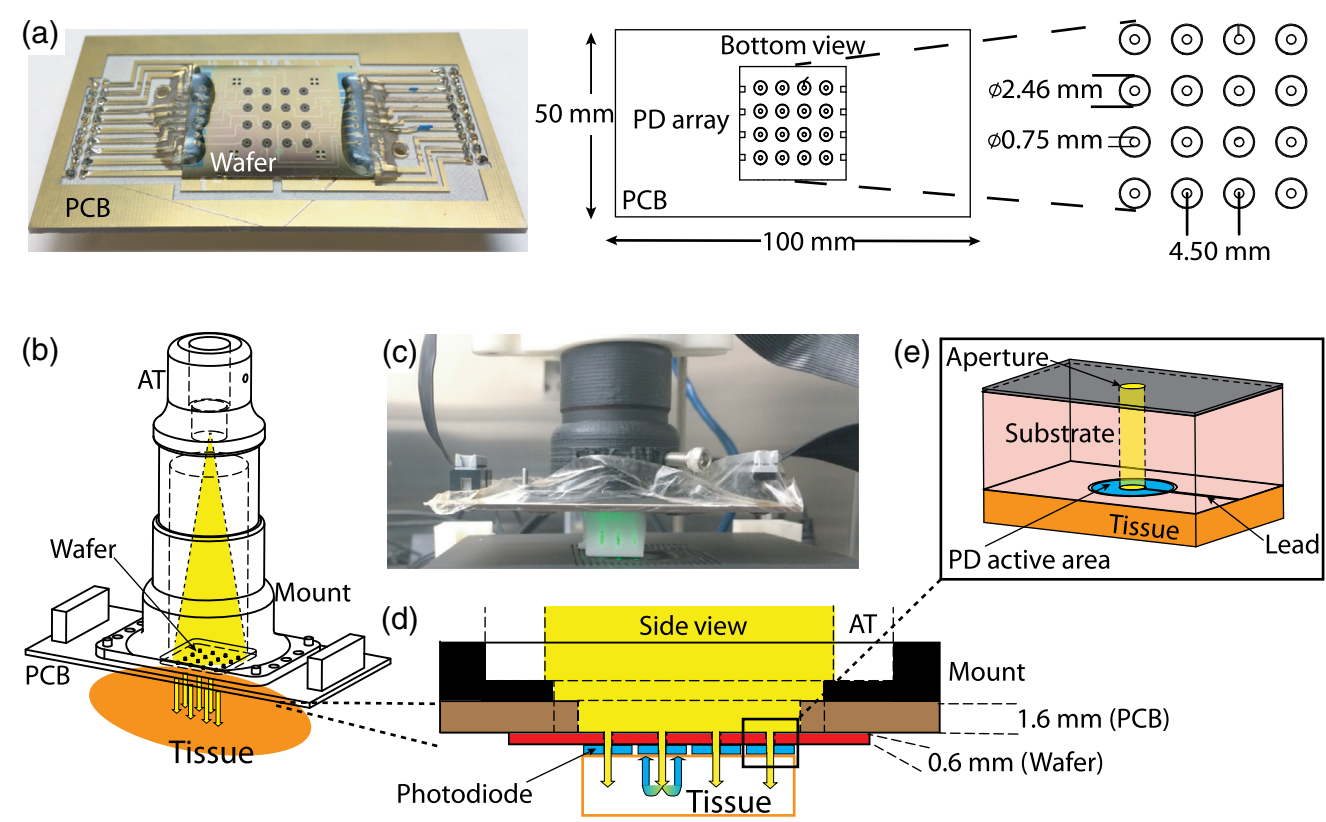

Fig. 2 Photodiode array design: (a) photo of board-mounted PDA from the bottom side with a corresponding diagram including dimensions and an expanded view of the photodiode layout, (b) illustration of light traversal from the LG to tissue, and (c) photo of the BMAP during acquisition on a foam resolution target. Note the near collimated beams within the sample emanating from the apertures. (d) A close-up cross-sectional view of the light traversal path including thickness dimensions of the board and silicon wafer. Note that the photodiode active area (shown in blue) has no thickness as these are just doped regions in the silicon wafer. Finally, (e) a close-up isometric view of a single photodiode as patterned on the silicon wafer. The leads for photocurrent readout are created by a metal evaporation process and have negligible thickness. 
to be greater than $35 \mathrm{~dB}$ for channels across all wavelengths, limited by the xenon lamp noise. The SNR was determined by acquiring 10 repeated measures on a $10 \%$ Spectralon $^{\mathrm{TM}}$ reflectance imaging target. The interpixel cross talk for the central pixels is $10 \%$ in the worst case scenario: tissue-specific cross talk is highest for specimens with albedo $\left[a=\mu_{\mathrm{s}}^{\prime} /\left(\mu_{\mathrm{s}}^{\prime}+\mu_{\mathrm{a}}\right)\right]$ near $1(\geq 0.97)$. Previous clinical studies conducted by our group indicate most breast tissue within an absorption range of (1.3 to $\left.20.4 \mathrm{~cm}^{-1}\right)$ and a scattering range of $\left(6.5\right.$ to $\left.11.3 \mathrm{~cm}^{-1}\right)$; average interpixel tissue cross talk is $\leq 4 \%$ in these ranges.

The board-mounted PDA is fastened to custom-made plastic acrylonitrile butadiene styrene (ABS) components designed to optimize free-space light delivery to the tissue (detailed in Sec. 2.4). The generalized light delivery and collection model is diagrammed in Fig. 2(b). As indicated in the figure, light is launched from a simple light guide (LG) at the distal end of an absorbing tube to the backside of the silicon PDA. Light simultaneously passes through each of the 0.75 -mm apertures to the probe-tissue interface on the PDA front side. When tissue is present, light enters and is multiply scattered or attenuated by absorption, a fraction of which will return to the front side of the PDA where it couples to one of the respective 16 annular photodiodes and is converted to a small electric current (photocurrent). The photocurrent is relayed to the transimpedance amplifier via two 20-pin ribbon cables, as seen in Fig. 2(c). A cross-sectional view of the photon path from source to detection is illustrated in Fig. 2(d), with an expanded view of a single annular photodiode diagrammed in Fig. 2(e).

\subsection{Photodiode Degradation}

Routine clinical use revealed substantial PDA deterioration marked by severe degradation of pixel responsivity. The use of germicidal cleaning agents and rubbing of the surface caused physical degradation of the antireflection coating and patterned metal contacts [Figs. 3(a) and 3(b)]. As the contacts were degraded, the effective photodiode active area was reduced, resulting in decreased optical response following each use. The abrasive cleaning technique was necessary due to tissue proteins and the rapid clotting of blood in direct contact with the PDA; removal of these substances required substantial physical force. To address this challenge, a thin $(60 \mu \mathrm{m})$ optically clear adhesive backed Teflon ${ }^{\mathrm{TM}}$ film was adhered to the PDA [Fig. 3(c)]. This coating did not interfere with the measured optical signal. An additional Tegaderm ${ }^{\mathrm{TM}}(3 \mathrm{M})$ layer was used to protect and waterproof the printed circuit board surrounding the PDA, as shown in Fig. 3(c). Note that the Tegaderm layer is hole-punched in the center to create a window for the imaging array. Utilization of the Teflon film was shown to have a negligible effect on extracted tissue optical properties;
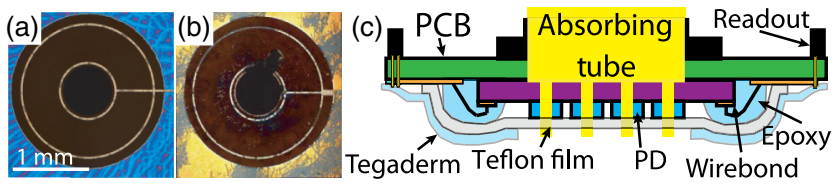

Fig. 3 Prevention of photodiode damage: (a) digital image of an unused PDA pixel, (b) image of a damaged PDA pixel, and (c) diagram of the protective Teflon and Tegaderm films. The Teflon film (thickness $=60 \mu \mathrm{m}$ ) is applied directly to the AR-coated PDA. A windowed Tegaderm film is layered over the entire surface of the probe bottom-side, leaving only the Teflon covered silicon exposed; this provides protection against biological compounds and cleaning agents. the measured optical intensity $[I(\lambda)]$ compared with and without Teflon had an root-mean-square deviation $<0.01$ and the extracted optical property accuracy remained the same (\%error $<10 \%)$. The influence of the Teflon film was determined in simulation and verified using the phantom study protocol discussed in Sec. 2.7. In addition to serving as a protective layer, the Teflon film was also shown to be reliably nonstick, effectively eliminating intermeasurement debris clinging to the probe from tissue contact. To validate this point, a clinical reproducibility study was performed wherein a single specimen was scanned three consecutive times $(n=10)$. On average, the interscan deviation was found to be $\sim 1 \%$.

\subsection{Light Source and Amplifier Design}

A $150 \mathrm{~W}$ Xenon lamp (Asahi Spectra, Japan) is coupled to an absorbing (matte black inner surface) spacer tube (ID = $30 \mathrm{~mm}$ ) using a hybrid LG $(\mathrm{NA}=0.57)$. The optimal length of the absorbing tube was determined to be $70 \mathrm{~mm}$ as this provided low cross talk (4\%) and nearly uniform illumination (center-pixel throughput ratio: 0.74). ${ }^{12}$ Aperture throughput ranged from 17 to $22 \mu \mathrm{W}$ per aperture while the total output from the hybrid LG ranged from 45 to $55 \mathrm{~mW}$. An 8-slot filter wheel within the Asahi lamp houses eight thin-film bandpass filters $(\mathrm{BP}=10 \mathrm{~nm})$ across the visible spectrum $(\lambda=$ [470480490500510560580600] nm). A custom mounting strategy is employed to minimize inadvertent user error and provide maximal throughput, as described in Appendix A.1.

A custom integrating transimpedance amplifier (ITIA) system was developed to convert the raw photocurrent to easily measured voltage that can be read by a laptop computer. The amplifier design consists of a precision (16-bit) USB data acquisition system (DAQ) (NI-USB-6212, National Instruments, Austin, Texas) containing 16 separate analog voltage inputs, as well as a custom-made low-noise 16-channel ITIA board (printed by Advanced Circuits, LLC). The smallest measurable change in intensity corresponds to a $\Delta \mathrm{IT}=10 \mu \mathrm{s}$ with a noise floor at $500 \mu \mathrm{s}$. Typically, an integration time of $50 \mathrm{~ms}$ was used for clinical measurements. An expanded description of the amplifier with circuit diagrams is shown in Appendix A.2.

\subsection{Imaging Platform}

A custom-made imaging platform was developed to provide robust quality control through automated positioning and probe handling. In addition to providing consistent contact pressure, the imaging platform is outfitted with stepper motor-controlled translational axes in each spatial dimension ( $x y z)$; this is utilized for subpixel sampling, implemented as a sequential raster scan. The imaging platform is diagrammed in detail in Appendix A.3. Pressure is measured as the total force applied to a custom-made copper base plate on which the tissue rests. The applied force is transduced by four discrete force sensors, each with a dynamic range of 1 to $44 \mathrm{~N}$, converted to pressure based on the area of the probe in contact with the tissue. A board-level USB camera is embedded as an interchangeable component and is utilized to capture digital images of the tissue for size estimation and coregistration of optical data with tissue locations marked with ink for histopathological review. Each of the translational axes is a custom-made leadscrew-based linear actuator designed to provide fine incremental motion yet move rapidly as to not significantly slow the raster-scanning 
acquisition process. Positional uncertainty (backlash) was shown to be $\leq 10 \mu \mathrm{m}$ upon direction reversal.

The probe is attached to the $z$-axis of the platform with a keyed circular clamp matched to the absorbing tube described in Sec. 2.4. The smallest $\Delta z$ was found to be $47 \mu \mathrm{m}$, corresponding to a pressure resolution $\Delta P=0.6 \mathrm{~mm} \mathrm{Hg}$ for the typical clinical specimen. Applied pressure and absolute position are coordinated by a dedicated embedded system to reduce PC computational load.

\subsection{Data Collection and Processing}

In clinical practice, the probe is incrementally translated in both lateral directions and subsequently translated downward to establish contact with the specimen resting on the pressure sensitive base. Within each placement, 16 independent measures of diffuse reflectance are captured via the 16 annular photodiodes. The reflectance as a function of wavelength is achieved by serially capturing the integrated intensity for each bandpass filter $\left[I(\lambda)_{s}\right]$, dividing this by the measured $I(\lambda)_{c}$ from a highly stable Spectralon lambertian surface $(\% R=99)$, and adjusting for the respective integration times. Before each clinical scan, a software-automated calibration sequence that measures the Spectralon at three integration times (IT $=25,50$, and $100 \mathrm{~ms}$ ) and three light intensity levels ( $\mathrm{LI}=50 \%, 75 \%$, and $100 \%)$ is executed; this sequence is used to decouple the source of day-today variations in measured Spectralon $I(\lambda)$ in addition to the wavelength-dependent source intensity. Intensity measurement calibration is then performed according to changes in the average slope of $I(\lambda, \mathrm{IT})$ and $I(\lambda, \mathrm{LI})$ as opposed to difference-based intensity levels. Note that a background measure is captured for each spectrum collected and is used to subtract stray light contributions from the intensity spectrum $\left[I(\lambda)_{s}=I(\lambda)-I(\lambda)_{\mathrm{bgd}}\right]$. The system response can vary due to light source degradation, changes in photodiode responsivity, scuffs on the Teflon film, and ambient conditions. The changes in system response found using the calibration scan are incorporated into the wavelengthdependent system scaling coefficients that were initially determined by the reference tissue phantom from the cross-validation tissue-simulating phantom study.

The result is a series of pressure bound multispectral images that are stitched into a high-resolution optical reflectance map $[R(\lambda, x, y, P)$, where $P$ is typically $10 \mathrm{~mm} \mathrm{Hg}]$. The highly reproducible pressure application at each point provides a reasonably uniform surface and preserves the optical characteristics of the ex vivo tissue. Each clinical raster-scan typically consists of 36 separate placements conducted in a zigzag pattern where the placement pitch is $0.75 \mathrm{~mm}$ in $x$ and $y$, resulting in 576 independent measures of reflectance for each $256 \mathrm{~cm}^{2}$ area, typically requiring $8 \mathrm{~min}$ or less depending on the specimen stiffness/elastic modulus. Each of the 576 reflectance spectra are run through a Monte-Carlo-based inverse model to obtain constituent optical property values $\left[\mu_{\mathrm{s}}^{\prime}(\lambda)\right.$ and $\left.\mu_{\mathrm{a}}(\lambda)\right]$, as described in Refs. 7, 13, and 14. Briefly, the inverse model utilizes a nonlinear least-squares fitting routine to best match a corrected reflectance spectrum to a Monte-Carlo-based reflectance lookup table established for the source-collection geometry and known optical interfaces. Absorber extinction spectra $[\varepsilon(\lambda)]$ are tabulated for the known constituent absorbers and are utilized as inversion priors, such that extracted absorber matrix coefficients represent concentration. Reflectance spectra are scaled prior to inversion by calibrating to a previously measured reference known as a "reference phantom," as described in Sec. 2.7.

\subsection{Homogeneous Optical Property Validation}

A set of 12 tissue-simulating phantoms was used to inform the homogeneous optical property extraction accuracy of the BMAP system. Each of the phantoms consisted of lyophilized human hemoglobin (Sigma-Aldrich Co. LLC, St. Louis, Missouri, $\mathrm{H} 7379$ ) and $1 \mu \mathrm{m}$ polystyrene microspheres (Polysciences, Inc., Warrington, Pennsylvania, 07310-15) as analogs for tissue absorption and scattering, respectively. The phantoms were mixed in proportions to span optical property ranges: $\left\langle\mu_{\mathrm{a}}\right\rangle_{\lambda}=0.5-5.5 \mathrm{~cm}^{-1}$ and $\left\langle\mu_{\mathrm{s}}^{\prime}\right\rangle_{\lambda}=2.9-9.5 \mathrm{~cm}^{-1}$ (averaged across all eight wavelengths used). The 12 phantoms were designed to subtend the 25 th to 75 th percentiles of $\left\langle\mu_{\mathrm{a}}\right\rangle_{\lambda}$ and $\left\langle\mu_{\mathrm{s}}^{\prime}\right\rangle_{\lambda}$ observed in breast tissue. The Monte-Carlo model accuracy for the BMAP system was assessed by evaluating the RMS errors between the extracted and expected phantom optical properties as described in Ref. 7. The specific optical properties and phantom constituents are listed in Table 1 . In addition to pipetting, the mass of each constituent was measured and recorded at each step of the phantom fabrication process to reduce discrepancies in optical property estimation from theoretical values. Each phantom was thoroughly mixed just prior to being measured to reduce the effects of solution settling. Ten repeated diffuse reflectance spectra were collected for all channels for each phantom. A leave-one-out cross-validation analysis, or "phantom study," was performed using each of the 12 tissue-simulating phantoms as a reference phantom against the remaining 11 . The reference phantom for clinical data inversion is selected by finding the phantom that, when used to scale the remaining 11 , results in the lowest error in estimating $\mu_{\mathrm{s}}^{\prime}(\lambda)$ and $\mu_{\mathrm{a}}(\lambda)$ for the entire phantom set. With the Spectralon calibration measurement, the reference phantom captures the system-specific response for the $R(\lambda) \rightarrow\left[\mu_{\mathrm{a}}(\lambda) \mu_{\mathrm{s}}^{\prime}(\lambda)\right]$ isomorphism. The multiphantom cross-validation technique is a robust approach that eliminates random user error as the entire Monte-Carlo lookup table is only scaled by the offset provided by a single best (reference) phantom such that improperly mixed phantoms and phantoms that do not adequately capture the system response can be ignored when constructing the scaling coefficient matrix. Note that the optical properties of the unused phantoms are still adequately predicted when scaled by the reference phantom, suggesting that the system response is linear although subject to artifacts on either end of the detector dynamic range.

\subsection{Resolution and Pressure Characterization}

The optimal scanning resolution was determined using a high contrast, custom resolution target [Fig. 4(a)] patterned atop a diffuse surface (dense white foam) to inform best-case scenario resolution achieved under clinically acceptable time constraints, typically 25 to $30 \mathrm{~min}$ at the Duke University Medical Center. The custom target was constrained by the probe footprint and foam rigidity; the very small protrusions made by the wirebonds from the silicon detector array to the printed circuit board would prevent total surface contact for any rigid object larger than $30 \times 30 \mathrm{~mm}$; thus, a $25.4 \times 25.4 \mathrm{~mm}$ target was fabricated. Furthermore features with, the target was optimized to only contain features with size scales relevant to the clinical needs of a margin assessment probe $(0.25$ to $3 \mathrm{~mm})$. The target was scanned at multiple raster-scan rates, hereafter represented 
Table 1 Homogenous liquid tissue simulating phantoms.

\begin{tabular}{lccccccccccrrr}
\hline \multicolumn{10}{c}{ Homogeneous phantom aliquot } \\
\hline Sample & 1 & 2 & 3 & 4 & 5 & 6 & 7 & 8 & 9 & 10 & 11 & 12 \\
\hline$\left\langle\mu_{\mathrm{s}}^{\prime}\right\rangle_{\lambda} \mathrm{cm}^{-1}$ & 3.98 & 4.41 & 4.93 & 5.44 & 5.94 & 6.44 & 6.95 & 7.45 & 7.96 & 8.47 & 8.99 & 9.45 \\
$\left\langle\mu_{\mathrm{a}}\right\rangle_{\lambda} \mathrm{cm}^{-1}$ & 0.16 & 0.82 & 1.18 & 1.54 & 1.91 & 2.26 & 2.61 & 2.97 & 3.34 & 3.71 & 4.08 & 4.47 \\
{$[\mathrm{Hb}] \mu \mathrm{M}$} & 2.65 & 13.6 & 19.6 & 25.5 & 31.5 & 37.3 & 43.2 & 49.1 & 55.2 & 61.4 & 67.5 & 73.7 \\
\hline
\end{tabular}

(a)

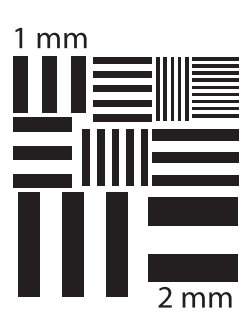

(b)

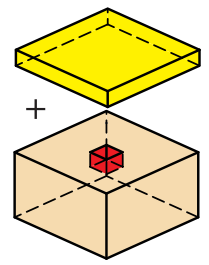

(c)

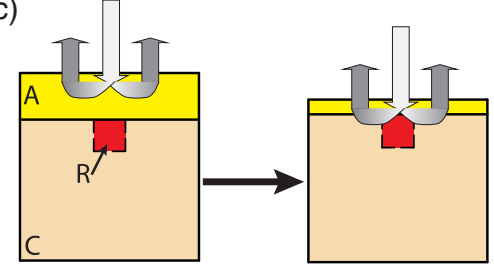

$25.4 \mathrm{~mm}$
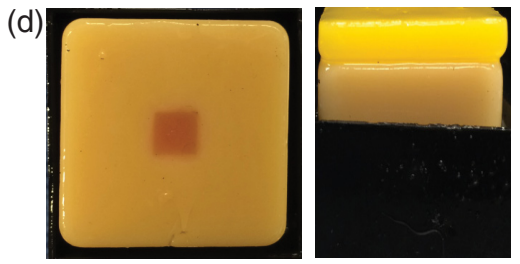

Fig. 4 Pressure and resolution experimental design: (a) the resolution target used in nonvolumetric, high-contrast resolution characterization for clinical $\Delta s$ optimization, (b) diagram of pressure experimental setup with layer and slab separated and (c) the anticipated effect as the pressure is increased; as the top layer is compressed, the distribution of photon paths include the cancer-like slab below the adipose layer. (d) Photographs of the physical phantoms used in the study; a top view of the slab and defect without the adipose layer is shown on the left and a side view of both the slab an layer (inside a container to maintain position) is shown on the right.

as sampling resolution in $\mathrm{mm}$ and referred to as $(\Delta s)$, and the time to complete the scan was automatically recorded for each scan.

While characterizing the resolution for high-contrast target helps optimize $\Delta s$, the question regarding the ability to resolve features with varying optical properties in tissue naturally arises. To characterize the volumetric, diffuse contrast (and, in a sense, resolution), three-dimensional (3-D) Monte-Carlo simulations were conducted for tissue slabs with contrasting perturbations of several sizes. These simulations were carried out using the same compressive sensing wavelengths leveraged by the BMAP system $(\lambda=[470480490500510560580600] \mathrm{nm})$. The Henyey-Greenstein phase function was assumed for $\mu_{\mathrm{s}}^{\prime}(\lambda)$. To incorporate the system response of the physical system in the simulated data, the phantom study reference phantom was also simulated; any wavelength-dependent differences between the simulated reflectance and actual reflectance can be used to scale all other simulated spectra. The large, semi-infinite slabs were designed to have optical properties that correspond to the median value of adipose tissue measured optical properties with our gold standard $49 \mathrm{ch}$ device (Table 2$)$. The dimensions of the slabs are $25.4 \times 25.4 \times 20 \mathrm{~mm}(l w h)$, with a perturbation region
Table 2 Tissue median values.

\begin{tabular}{lccc}
\hline Sample & Adipose & Reference phantom & Cancer \\
\hline$\left\langle\mu_{\mathrm{s}}^{\prime}\right\rangle_{\lambda} \mathrm{cm}^{-1}$ & 7.12 & 6.78 & 13.7 \\
$\left\langle\mu_{\mathrm{a}}\right\rangle_{\lambda} \mathrm{cm}^{-1}$ & 3.99 & 1.39 & 3.78 \\
{$[\mathrm{Hb}] \mu \mathrm{M}$} & 15.28 & 22.7 & 30.9 \\
{$[\beta-\mathrm{C}] \mu \mathrm{M}$} & 19.58 & 0.00 & 12.98 \\
\hline
\end{tabular}

in the center of the slab that extends $3 \mathrm{~mm}$ in depth and has an en face square surface with $l=w=[0.25,0.75,1.5] \mathrm{mm}$. Similarly, the optical properties of the perturbation were designed to match the median measured values of invasive carcinoma tissue (cancer) (Table 2). To adequately characterize the diffuse contrast, simulations were carried out for $\Delta s=$ $[0.25,0.75,1.5,4.5] \mathrm{mm}$ for each perturbation size.

Clinically relevant pressure ranges were informed using layered gelatin-based $(60 \mathrm{mg} / \mathrm{mL})$ solid tissue-simulating phantoms optimized to have a stress/strain ratio (Youngs Modulus) comparable to that observed during routine clinical use $(0.08$ to $11.3 \mathrm{kPa}$ ) and similar to those reported in literature. ${ }^{15}$ Note that because the imaging platform tracks the $x y z$ position for each measurement, tissue compression (via probe height) can be captured as a function of pressure during each clinical measurement. Solid phantom slabs were designed to simulate clinically relevant tissue geometry and optical properties: a 2-mm-thick slab was designed to have optical properties corresponding to purely adipose tissue to simulate a negative margin geometry; the 2 -mm layer was placed on top of a larger $25.4 \times 25.4 \times 20$ $(l \times w \times h) \mathrm{mm}$ slab with optical properties correlated to invasive breast cancer. To corroborate changes due to pressure, the cancerous slab has an embedded defect $(4.5 \times 4.5 \times 4.5 \mathrm{~mm})$ with optical properties designed to match the reference phantom to provide a secondary source of contrast beneath the adipose layer [Fig. 4(b)]. The corresponding optical properties were informed using optical parameter distributions from previously acquired data for each tissue subtype (histopathologically verified by a practicing clinical pathologist). Note that the optical properties for the constructed solid phantoms deviate slightly from those in Table 2; the difference is due to mixture uncertainty and intentional enhancement of contrast. As a result, the cancer-like tissue has increased $\left\langle\mu_{\mathrm{s}}^{\prime}\right\rangle_{\lambda}$ and decreased $[\beta-$ carotene], while the adipose tissue has further increased $[\beta$ carotene] and decreased [Hb]. A summary of the final optical properties can be observed in Table 3. To understand the effect of pressure on extracted optical properties, the layered sample 
Table 3 Solid gelatin tissue phantoms.

\begin{tabular}{lccc}
\hline \multicolumn{4}{c}{ Solid phantom components } \\
\hline Sample & $\begin{array}{c}2 \text { mm layer } \\
\text { (adipose) }\end{array}$ & $\begin{array}{c}\text { Heterogeneity } \\
\text { (reference) }\end{array}$ & $\begin{array}{c}\text { Slab } \\
\text { (cancer) }\end{array}$ \\
\hline$\left\langle\mu_{\mathrm{s}}^{\prime}\right\rangle_{\lambda} \mathrm{cm}^{-1}$ & 8.67 & 6.64 & 16.38 \\
$\left\langle\mu_{\mathrm{a}}\right\rangle_{\lambda} \mathrm{cm}^{-1}$ & 2.49 & 1.11 & 1.54 \\
{$[\mathrm{Hb}] \mu \mathrm{M}$} & 2.51 & 16.7 & 16.7 \\
{$[\beta-\mathrm{C}] \mu \mathrm{M}$} & 24.1 & 0.00 & 4.45 \\
{$[\mathrm{Gel}]$} & 60.0 & 55.9 & 59.3 \\
$\mathrm{mg} / \mathrm{mL}$ & & &
\end{tabular}

was raster-scanned 12 times corresponding to pressures $P=[3,6,9,12,15,18,21,25,33,37,43,49] \mathrm{mm} \mathrm{Hg}$. Similar to clinical use, the layered sample was raster-scanned with $\Delta s=$ $0.75 \mathrm{~mm}$ (36 placements) using the zigzag pattern previously mentioned. The experimental design and hypothesized effect are outlined in Fig. 4(c). Photographs of the solid phantoms used in the study are shown in Fig. 4(d); the last photograph demonstrates the phantom under load. To preserve the integrity of the solid phantoms, each component was immersed in foodgrade mineral oil when not in use once completely cured. The immersion in mineral oil prevents moisture from escaping the phantoms and drying them out, unpredictably changing the optical properties. Note that because collagen (in gelatin) is not soluble in any oil, or any of the phantom constituents, this immersion also prevents substantial penetration of the oil within the phantom. An additional benefit of this immersion is the prevention of bacterial or fungal growth; the phantoms do not mold or spoil over time. The major drawback of this immersion is the lack of oxygen available to the phantoms, necessarily causing oxy-hemoglobin to convert to deoxy-hemoglobin over time. To suspend the phantom oxygenation state, formalin $(1 \mathrm{~mL}$, $10 \%$ solution) was immersed into the phantoms immediately after curing (initial water volume was adjusted to account for the added formalin), causing the hemoglobin protein to become fixed in an oxygenated state. The phantoms were cast in a two stage process. First the phantoms were cast in a mold with a protrusion based on the size of the defect, resulting in a homogeneous slab with a hole where the defect should be. A thin layer of mineral oil was added to the exposed surfaces to prevent unwanted mixing and loss of moisture. The defect (hole) was then filled with the reference phantom-like gelatin-based solution and allowed to cure, with the formalin added immediately after and allowed to diffuse within the sample completely. After formalin diffusion, the completed phantom was immersed in mineral oil and stored at $4^{\circ} \mathrm{C}$ until used. The optical properties for the solid phantoms were measured randomly during a 1month period, and no detectable changes (deviations $>5 \%$ ) were observed. Finally, due to the fact that $\beta$-carotene is insoluble in water, Crocin (Sigma-Aldrich Co. LLC, St. Louis, Missouri) was used as an analog.

\subsection{Clinical Data Collection}

Diffuse reflectance spectra were collected from excised breast tissue specimens from a total of three margins in three separate patients for the proof-of-concept data shown in this paper. This study was performed in strict accordance with Duke University Institutional Review Board protocol: Pro00028284. Patients, who were 18 years and older and having the lumpectomy procedure, granted written consent under the approved protocol. Specimen orientation for lumpectomies (partial mastectomies) was determined according to surgically placed reference features including: a surgical wire inserted into the center of the tumor, colored sutures, and surgical clips. Specimen faces were defined as the faces of a cube and labeled relative to the specimen orientation in situ; the six measurable faces are hereafter referred to as the superior, inferior, posterior, anterior, medial, and lateral margins. Immediately following tissue resection, partial mastectomy specimens were sent to radiology for an intraoperative mammography to verify removal of the tumor mass. Upon return, the specimen was placed on the pressuresensing base of the imaging platform and oriented accordingly. Following orientation, the raster-scanning procedure was initiated and the diffuse reflectance spectra were collected. Once the scanning procedure was completed, a site-level inking procedure was performed wherein 6 to 10 sites were randomly marked using tattoo ink (typically orange in color) with the aid of a coregistration structure that physically relayed the central location of each optical channel to the respective point of contact with the specimen. A single margin was inked for postoperative pathological assessment. Margin inking was followed by the acquisition of a digital image using the previously mentioned (Sec. 2.5) USB digital camera assembly. Tissue optical property maps were reconstructed postmeasurement using the inverse Monte-Carlo model discussed previously. To establish optical extraction equivalence to previous generation devices, the samples in this work were measured using both the BMAP system and our gold-standard 49ch system described in Sec. 1 and Ref. 16. These measurements were performed by swapping the probes only; the tissue specimen was not moved or realigned. The on-board positioning tracking of the imaging platform was used to determine the corresponding regions measured by each probe, which share a central optical axis as mentioned in Sec. 2.5.

\section{Results}

\subsection{Optical Property Extraction Accuracy}

Using the seventh (middle) phantom $\left(\left\langle\mu_{\mathrm{a}}\right\rangle_{\lambda}=3.5,\left\langle\mu_{\mathrm{s}}^{\prime}\right\rangle_{\lambda}=\right.$ $6 \mathrm{~cm}^{-1}$ ) as a scaling reference, the average percent error across all phantoms and pixels in extracting $\mu_{\mathrm{s}}^{\prime}$ and $\mu_{\mathrm{a}}$ was found to be $5.69 \pm 1.88 \%$ and $8.91 \pm 1.72 \%$, respectively. The extracted phantom values are plotted against the expected values in Fig. 5. These error ranges are comparable to our current gold standard device, as shown in Ref. 16. The deviation of the reference phantom measured reflectance from the expected reflectance produced by the forward Monte-Carlo model is used to calibrate all measured spectra for inversion and accounts for the system response not captured by the forward model, as described in Ref. 7. Note that because the PDA geometry captures light near the input source, the $\mu_{\mathrm{s}}^{\prime}(\lambda)$ is sensitive to the scattering phase function; this is discussed in additional detail in Sec. 4.

\subsection{Resolution Characterization}

From a clinical timing perspective, an upsample factor of 6 or $\Delta s=0.75 \mathrm{~mm}$ proved to be ideal; additional gains in twodimensional (2-D) feature resolution beyond this $\Delta s$ were 


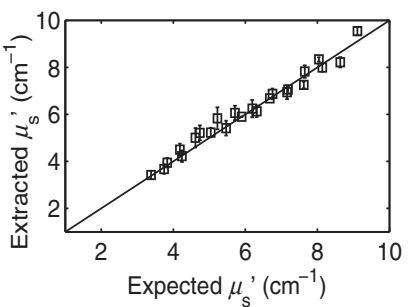

(a)

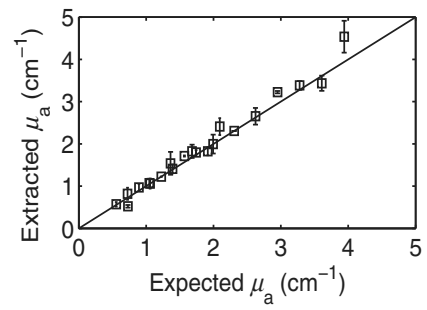

(b)
Fig. 5 Homogeneous optical property extraction error: (a) average percent error in $\mu_{\mathrm{s}}^{\prime}$ and (b) average percent error in $\mu_{\mathrm{a}}$. The diagonal line indicates perfect agreement. Error bars represent the standard deviation of the extracted values across pixels. Wavelength averaged $\mu_{\mathrm{s}}^{\prime}$ and $\mu_{\mathrm{a}}$ for the phantom used as a reference were 6 and $3.5 \mathrm{~cm}^{-1}$, respectively. Note that the indicated error includes the use of the Teflon film mentioned in Sec. 2.2.

minimal due to the large illumination apertures $(0.75 \mathrm{~mm})$, while the total scan time was substantially increased (9.61 to $13.1 \mathrm{~min}$ ) [Fig. 6(a)]. Note that because the target is not completely opaque (there is some diffusion of light), the feature resolution does not strictly follow the Whittaker-Shannon sampling theorem; for a $\Delta s=0.75 \mathrm{~mm}, 1 \mathrm{~mm}$ features are adequately resolved without aliasing.

In tissue, the smallest cancer-like heterogeneity that we can reasonably expect to detect and correctly classify is roughly $0.75 \mathrm{~mm}$ for a $\Delta s=0.75 \mathrm{~mm}$. As shown in Fig. 6(b), for $\Delta s=0.75 \mathrm{~mm}$, the cancer-like heterogeneity has enough contrast against the background (adipose-like tissue) to manifest as an area with $[\beta$-carotene $] /\left\langle\mu_{\mathrm{s}}^{\prime}\right\rangle_{\lambda} \leq 2$, the utilized threshold differentiating likely cancer from likely normal tissue in this work. Furthermore, the benefit of subpixel sampling via raster-scanning is clearly presented in Fig. 6(b); the uncertainty (indicated by the error bars) in the heterogeneity size, position, and optical property values decreases nonlinearly with decreasing $\Delta s$. At the native probe resolution $(\Delta s=4.5 \mathrm{~mm})$, it is only possible to correctly classify the largest heterogeneity $(\Delta b=1.5 \mathrm{~mm})$, and the likelihood of doing so is only $33 \%$.

For the purposes of detecting residual disease at the tumor margin, it is sufficient to merely detect its presence in a binary sense; the precise position, optical parameter value, and size are not critical to be determined as long as positive margins are not missed. An expanded view of the expected contrast in tissue is shown in Figs. 7(a)-7(c). The possible values in estimating $[\beta$-carotene $] /\left\langle\mu_{\mathrm{s}}^{\prime}\right\rangle_{\lambda}$ for three separate sized cancer-like heterogeneities can be seen as a function of both $\Delta s$ and probe position $(\chi)$ for a single en face line scan across the heterogeneity. The cancerous heterogeneities and adipose slabs have a true value of $[\beta$-carotene $] /\left\langle\mu_{\mathrm{s}}^{\prime}\right\rangle_{\lambda}=0.9$ and $[\beta$-carotene $] /\left\langle\mu_{\mathrm{s}}^{\prime}\right\rangle_{\lambda}=$ 2.97, respectively, and the gray plane represents the $[\beta$-carotene $] /\left\langle\mu_{\mathrm{s}}^{\prime}\right\rangle_{\lambda}=2$ threshold utilized in this paper. As the figure shows, the $0.25-\mathrm{mm}$ heterogeneity $(\Delta b=0.25 \mathrm{~mm})$ [Fig. 7(a)] cannot be resolved with the current PDA geometry, even if sampled at $\Delta s=0.25 \mathrm{~mm}$. For the $0.75-\mathrm{mm}$ heterogeneity $(\Delta b=0.75 \mathrm{~mm})$ in Fig. $7(\mathrm{~b})$, it can be seen that there is only a small subregion of $\Delta s$ that can adequately differentiate the heterogeneity from the adipose background (the region below the gray plane). The effect of $\Delta s$ on the possible estimated $[\beta$-carotene $] /\left\langle\mu_{\mathrm{s}}^{\prime}\right\rangle_{\lambda}$ values is condensed in a box-andwhisker style plot in Figs. 7(e) and 7(f). (a)

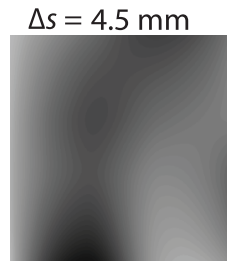

$t=0.27 \mathrm{~min}$

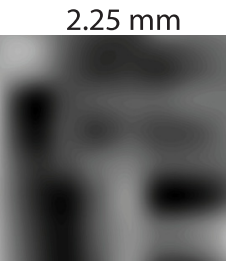

$1.06 \mathrm{~min}$

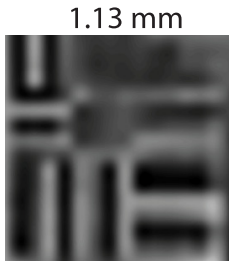

$4.27 \mathrm{~min}$

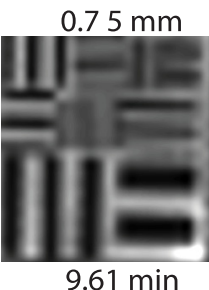

$9.61 \mathrm{~min}$

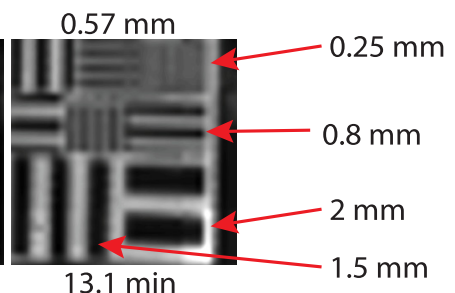

$13.1 \mathrm{~min}$

(b)

$\therefore \Delta \mathrm{b}=0.25 \times 0.25 \mathrm{~mm}$ $\begin{aligned} \square & b=0.75 \times 0.75 \mathrm{~mm} \\ \Delta \mathrm{b} & =1.5 \times 1.5 \mathrm{~mm}\end{aligned}$
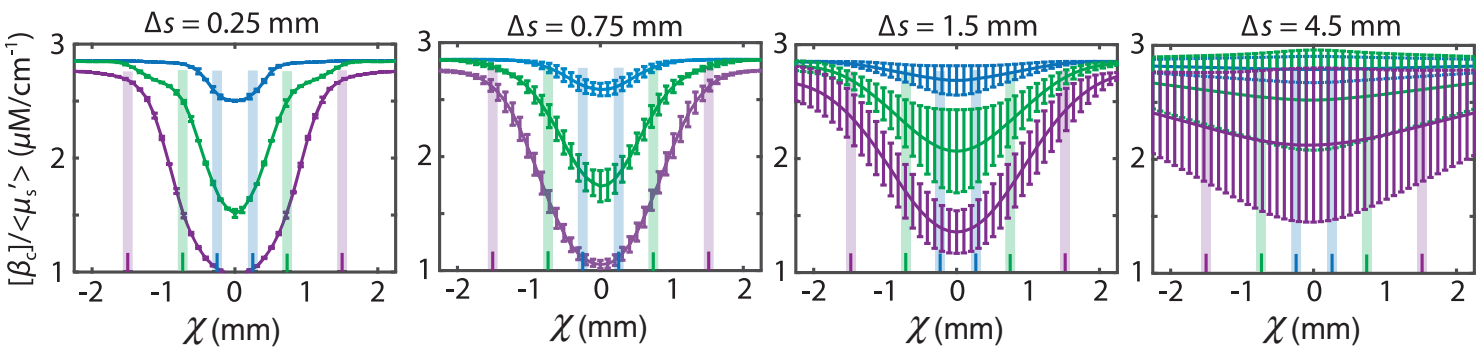

Fig. 6 BMAP resolution characterization: (a) Reflectance measured on a high contrast resolution target for a series of raster-scan rates with increasing $\Delta s$ from left to right. The time required for each scan is indicated below the respective image and (b) line scans across simulated diffuse, tissue-like slabs each with an embedded heterogeneity. The center position of the simulated photodiode is represented by $\chi$. Within each plot, line scans are shown as the extracted $[\beta$-carotene $] /\left\langle\mu_{\mathrm{s}}^{\prime}\right\rangle_{\lambda}$ for three heterogeneity sizes $(\Delta b=[0.50,0.75,1.5] \mathrm{mm})$. Heterogeneities are centered at $\chi=0$. From left to right, $\Delta s$ is increased from 0.25 to $4.5 \mathrm{~mm}$, depicting the loss of contrast for each heterogeneity as a function of sampling rate. The error-bars represent all possible $[\beta$-carotene $] /\left\langle\mu_{\mathrm{s}}^{\prime}\right\rangle_{\lambda}$ estimates (uncertainty) derived from the inverse model for the simulated spectra. 

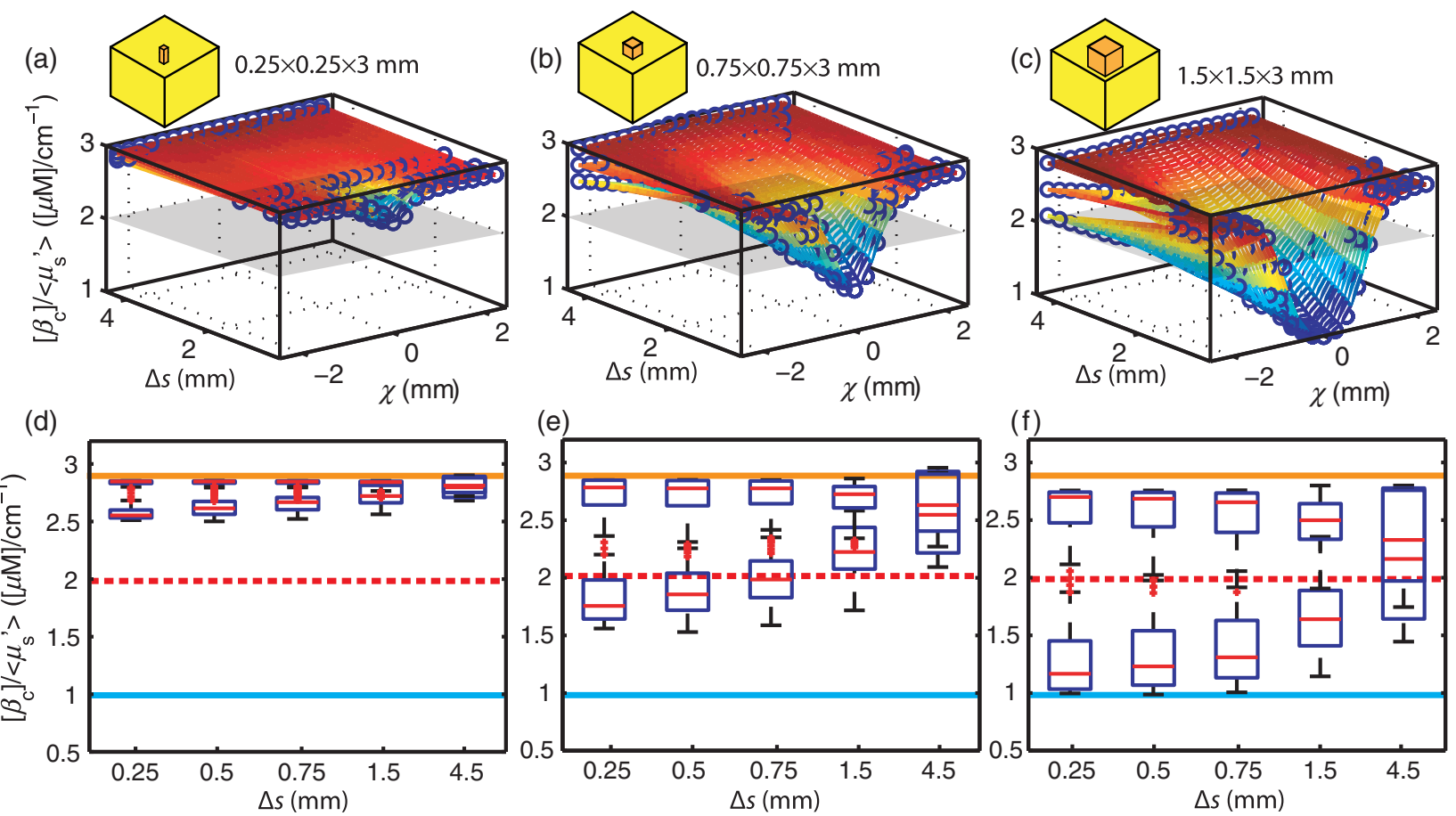

Fig. 7 Contrast in tissue. (a) Possible $[\beta$-carotene $] /\left\langle\mu_{\mathrm{s}}^{\prime}\right\rangle_{\lambda}$ values for a line scan $(\chi)$ across a rectangular volume $(I \times w \times h=0.25 \times 0.25 \times 3 \mathrm{~mm})$ with cancer-like optical properties surrounded by a semi-infinite adipose slab (the geometry is shown in Sec. 2.8), shown as a function of $\Delta s$. Similarly, (b) and (c) represent possible $[\beta$-carotene $] /\left\langle\mu_{\mathrm{s}}^{\prime}\right\rangle_{\lambda}$ values for perturbations in increasing size $(0.75$ and $1.5 \mathrm{~mm}$, respectively). The center position of the simulated photodiode is represented by $\chi$. The gray plane represents the threshold for which the heterogeneity would be correctly classified as "likely cancer." In (d) through (f), the possible $[\beta$-carotene $] /\left\langle\mu_{\mathrm{s}}^{\prime}\right\rangle_{\lambda}$ estimates are condensed in box-and-whisker plot format. The blue line represents the true $[\beta$-carotene $] /\left\langle\mu_{\mathrm{s}}^{\prime}\right\rangle_{\lambda}$ value for the cancer-like heterogeneity and the expected median value for the lower row of boxes. The dashed red line indicates the threshold for which the heterogeneity would be correctly diagnostically classified. The orange line represents the true $[\beta$-carotene] $] /\left\langle\mu_{\mathrm{s}}^{\prime}\right\rangle_{\lambda}$ value for the adipose slab and the expected median value for the upper row of boxes.

\subsection{Effects of Pressure}

The pressure applied at the probe-tissue interface was observed to cause substantial deviations in optical parameter estimates for the solid tissue phantoms, as described in Sec. 2.8. As shown in Fig. $8(\mathrm{a})$, the estimated $[\beta$-carotene $]$ at pressures below $5 \mathrm{~mm}$ $\mathrm{Hg}$ was observed to be wildly inconsistent, suggesting insufficient or partial contact with the layered phantom; however, at pressures $\geq 15 \mathrm{~mm} \mathrm{Hg}$, the estimates for [ $\beta$-carotene] begin to decrease as the 2-mm layer begins to compress and the slab below is included in the photon path; both regions below the adipose layer contain much less of the $\beta$-carotene analog (Crocin). A similar effect was observed for $\left\langle\mu_{\mathrm{s}}^{\prime}\right\rangle_{\lambda}$ and is shown in Fig. 8(b). The optical changes suggest a window where optical property fidelity is maintained, roughly between 6 and $15 \mathrm{~mm} \mathrm{Hg}$. Thus, for routine clinical use, a pressure of $10 \mathrm{~mm} \mathrm{Hg}$ was used.

The physical amount of compression was observed to be nearly $10 \mathrm{~mm}$ for the greatest pressures used $(\geq 15 \mathrm{~mm} \mathrm{Hg})$ [Fig. 8(c)]. Distributions of $[\beta$-carotene $] /\left\langle\mu_{\mathrm{s}}^{\prime}\right\rangle_{\lambda}$ are shown in Figs. $8(\mathrm{~d})$ and $8(\mathrm{e})$ for $4.5 \times 4.5 \mathrm{~mm}$ regions of the parameter maps in (a) and (b), indicated as box (1) and (2), corresponding to areas of the large phantom slab containing optical properties representing the reference phantom (low $\left\langle\mu_{\mathrm{s}}^{\prime}\right\rangle_{\lambda}$ and no [ $\beta$-carotene]) and cancerous tissue (high $\left\langle\mu_{\mathrm{s}}^{\prime}\right\rangle_{\lambda}$ and low [ $\beta$-carotene]), respectively. As seen in $8(\mathrm{e})$, the $[\beta$-carotene $] /\left\langle\mu_{\mathrm{s}}^{\prime}\right\rangle_{\lambda}$ values drop below the actual value (2.97) at pressures beyond $15 \mathrm{~mm} \mathrm{Hg}$, suggesting that the "clear" margin would not be read as clear adipose tissue, but perhaps with some fibroglandular content.

\subsection{Clinical Validation}

Clinical specimens (three total) measured using the BMAP system were found to exhibit similar optical parameter distributions to those captured with the gold standard $49 \mathrm{ch}$ system and were consistent with our previously published results. ${ }^{8,9,16}$ The clinical utility of the BMAP on a representative clinical specimen with a close but negative margin is shown in Figs. 9(a)-9(d). The labeled sites correspond to histopathology-verified sitelevel data reviewed by an expert pathologist. The high-resolution parameter maps [Fig. 9(d)] reveal a level of detail that is not apparent in the low-resolution reflectance image [Fig. 9(c)], demonstrating the importance of subpixel sampling. Congruent with previously published results, areas corresponding to fibroglandular tissue (region 3) have markedly lower $[\beta$-carotene $] /\left\langle\mu_{\mathrm{s}}^{\prime}\right\rangle_{\lambda}$ values compared to regions of adipose tissue (region 4). Note that this stark contrast is lost in the low-resolution parameter map [Fig. 9(c)]. Region 5 corresponds to a site with invasive ductal carcinoma $1.8 \mathrm{~mm}$ distal (in depth) to the margin surface; the entire region contains markedly lower $[\beta$-carotene $] /\left\langle\mu_{\mathrm{s}}^{\prime}\right\rangle_{\lambda}$ values relative to other sites; this is aligned with a generally accepted notion of stromal modification near malignant sites. ${ }^{17-19}$ The mammographic breast density (MBD) for the sample was reported to be high $(\mathrm{MBD}=4)$. MBD scores 

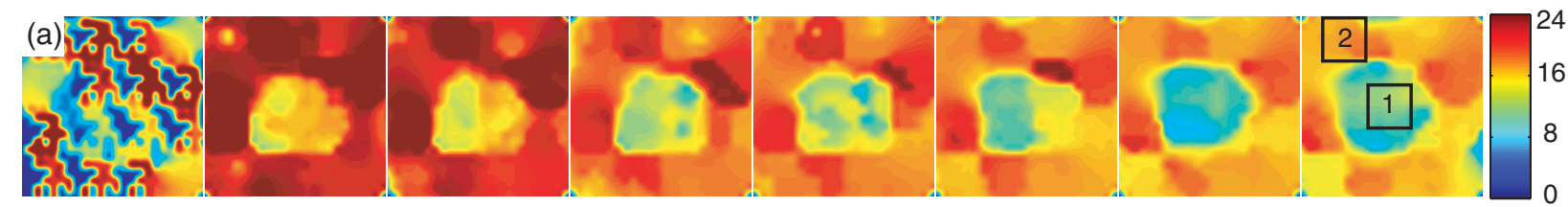

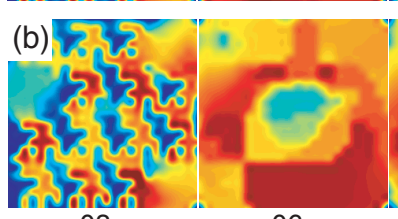

03

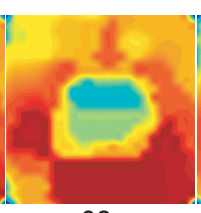

09

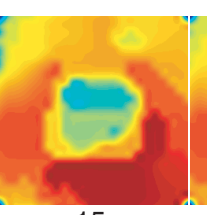

15

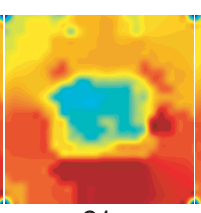

21

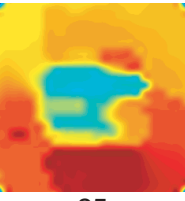

25

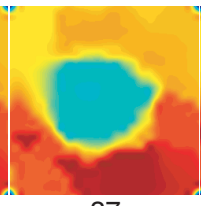

37

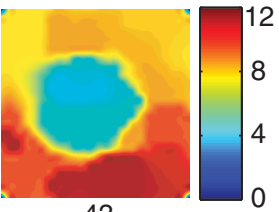

43
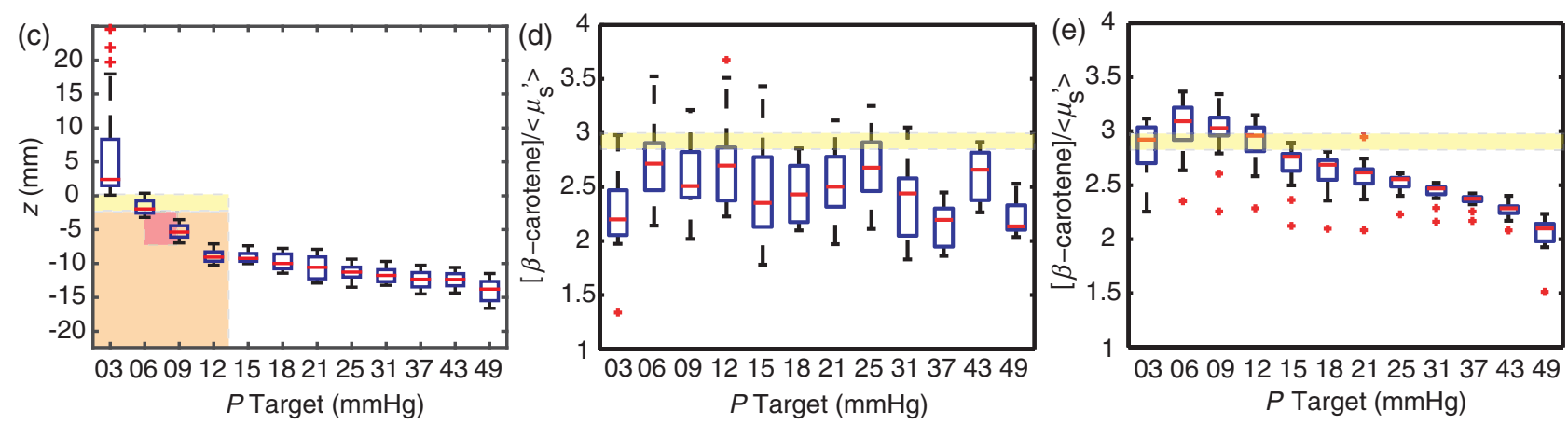

$P$ Target $(\mathrm{mmHg})$

Fig. 8 Pressure-induced optical parameter deviations. (a) Map of [ $\beta$-carotene] concentration of the layered phantom with a two region slab [high scattering low beta, low scattering low beta (center region)]. Likewise, (b) shows the wavelength averaged $\mu_{\mathrm{s}}^{\prime}\left(\mathrm{cm}^{-1}\right)$ and reveals a similar trend as the center region has a slightly reduced $\left\langle\mu_{\mathrm{s}}^{\prime}\right\rangle_{\lambda}$ (7 to 6.2). (c) Shows the amount of compression during the experiment with the phantom diagrammed in the plot; yellow represents the adipose layer, red the center region, and orange the remaining cancer slab. (d) Shows the $[\beta$-carotene $] /\left\langle\mu_{\mathrm{s}}^{\prime}\right\rangle_{\lambda}$ trend for the center region indicated by the box labeled 1 . The yellow line represents the expected value. (e) Similarly shows the trend for the region indicated by box 2 and not influenced by the center region. Note the distinct drop in the $[\beta$-carotene $] /\left\langle\mu_{\mathrm{s}}^{\prime}\right\rangle_{\lambda}$ ratio beyond $15 \mathrm{~mm} \mathrm{Hg}$; this is due to the highly scattering nature of the cancer slab, having a $\left\langle\mu_{\mathrm{s}}^{\prime}\right\rangle_{\lambda}$ of $15 \mathrm{~cm}^{-1}$.

are indicated as 1-fatty, 2-scattered fibrous tissue, 3-heterogeneously dense, and 4-extremely dense. High MBD has a known association with increased breast cancer risk, particularly in premenopausal patients. ${ }^{20,21}$ Figures $9(\mathrm{e})-9$ (h) show an additional clinical specimen with a high $\mathrm{MBD}=3$. Note the overall lower distribution of $[\beta$-carotene $] /\left\langle\mu_{\mathrm{s}}^{\prime}\right\rangle_{\lambda}$ values, which again corroborates significant stromal involvement for dense, fibrous regions of tissue due to extensive collagen involvement.

The similarity to the 49ch gold-standard device is also demonstrated in Figs. 9(e)-9(h). Figure 9(f) shows a full margin scan captured with the 49ch device of a mammographically dense breast $(\mathrm{MBD}=3$ ); the region measured by both devices is indicated by the dashed black box. A zoomed and cropped 49ch parameter map of the black box region is shown in Fig. 9(g); note the similarity to the same parameter map captured with the BMAP system shown in Fig. 9(h). A similar representation is shown on a low-density breast $(\mathrm{MBD}=2)$ in Figs. 9(i)-9(1). As a corollary to the previously mentioned hypothesis, the overall increased $[\beta$-carotene $] /\left\langle\mu_{\mathrm{s}}^{\prime}\right\rangle_{\lambda}$ values indicate reduced fibroglandular extent and a greater percentage of fatty tissue; indeed the measurement distribution shown here results from both increased [ $\beta$-carotene] and decreased $\left\langle\mu_{\mathrm{s}}^{\prime}\right\rangle_{\lambda}$.

A summary of the extracted $[\beta$-carotene $] /\left\langle\mu_{\mathrm{s}}^{\prime}\right\rangle_{\lambda}$ values for each of the three margins is shown in Fig. $9(\mathrm{~m})$ as CDFs. For each margin, an additional CDF corresponding to the same region measured with our gold standard 49ch device is overlaid to demonstrate the equivalence in extracted optical parameter value distributions. The similarity of these optical parameter distributions is alternatively quantified and is shown in the form of box-and-whisker plots in Fig. 9(n).

\section{Discussion}

Presented in this paper is a margin assessment system designed to address some of the key challenges in breast tumor margin assessment: detecting residual disease at the time of surgery and providing a scalable platform that lends itself to clinical adoption. Performance-wise, the BMAP system strikes an FOV, resolution, and speed balance on par with research-grade instruments in a low-cost package. Indeed, our group's current 49ch QDRI clinical system consists of an astronomy grade CCD/ spectrograph and a bulky yet delicate fiber-optic probe. We have previously shown that the clinical QDRI technology can accurately survey breast tumor margins rapidly with reasonable sensitivity and specificity. ${ }^{8}$ The problem with previous generation devices stems from the difficulty in scaling the high-precision, research-grade components; there is no obvious path for commercialization and mass-production, suggesting widespread clinical adoption would be challenging and unlikely. Here, we sought to develop a pragmatic, low-cost, bare-essentials system; these goals are the primary reason that almost every system component is custom-made or an extremely basic low-cost integrated circuit (IC) or hardware component. The 3-D-printed plastic pieces and off-the-shelf parts easily scale for massproduction via injection molding, volume ordering, and simple 

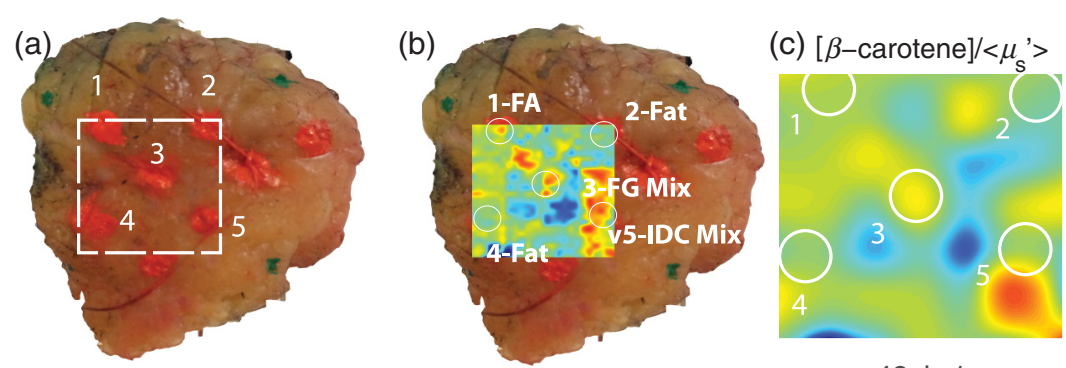

BMAP-2
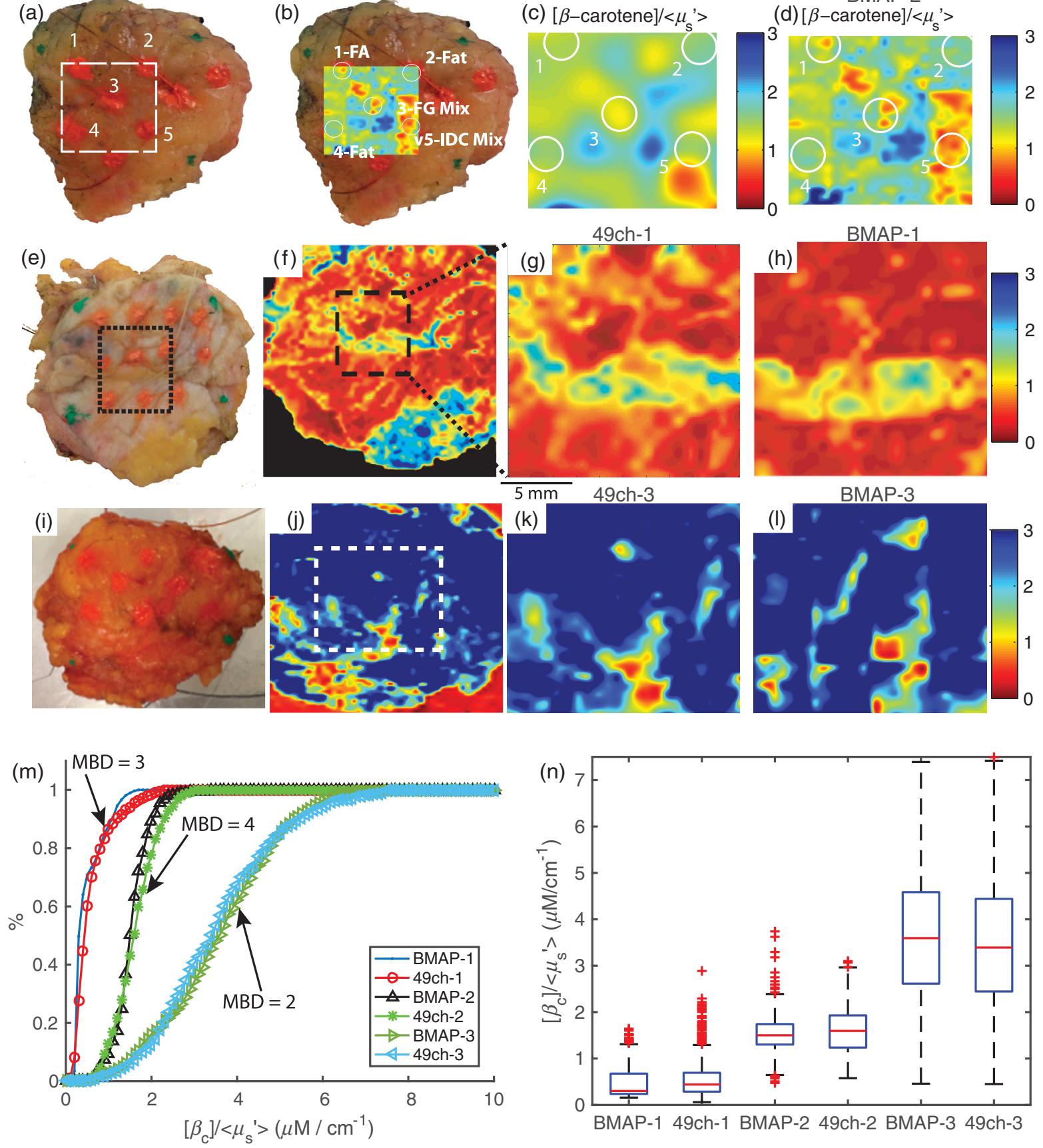

Fig. 9 Clinical margin data: (a) digital image of a close margin (BMAP-2) showing sites inked for pathological review, (b) digital image with $[\beta$-carotene $] /\left\langle\mu_{\mathrm{s}}^{\prime}\right\rangle_{\lambda}$ map overlayed, (c), $[\beta$-carotene $] /\left\langle\mu_{\mathrm{s}}^{\prime}\right\rangle_{\lambda}$ map with $\Delta s=4.5 \mathrm{~mm}$, and (d), $[\beta$-carotene $] /\left\langle\mu_{\mathrm{s}}^{\prime}\right\rangle_{\lambda}$ map with $\Delta s=0.75 \mathrm{~mm}$ with outlines corresponding to inked regions. FA, fibro-adipose; FG, fibroglandular; and IDC, invasive ductal carcinoma. Similarly, (e) a digital image of an additional close but negative margin with (f) the corresponding wide-field [ $\beta$-carotene $] /\left\langle\mu_{\mathrm{s}}^{\prime}\right\rangle_{\lambda}$ map acquired with the $49 \mathrm{ch}$ gold-standard clinical system. The black dashed box indicates the region also measured by the BMAP system. (g) A cropped and zoomed map of the coregistered region captured with the 49ch device (49ch-1) and (h) the corresponding $[\beta$-carotene $] /\left\langle\mu_{\mathrm{s}}^{\prime}\right\rangle_{\lambda}$ map captured with the BMAP device (BMAP-1). In (i) through (I), the digital, 49ch full FOV, 49ch-cropped (49ch-3), and corresponding BMAP parameter map (BMAP-3) are shown for a low-density margin $(\mathrm{MBD}=2)$. In $(\mathrm{m})$, the $[\beta$-carotene $] /\left\langle\mu_{\mathrm{s}}^{\prime}\right\rangle_{\lambda}$ cumulative distributions for each of the 49ch/BMAP pairs with the mammographic breast density (MBD) indicated. Finally, (n) the $[\beta$-carotene $] /\left\langle\mu_{s}^{\prime}\right\rangle_{\lambda}$ distributions of the coregistered regions shown in a condensed box-and-whisker format.

assembly line style construction; this approach requires nothing special of manufacturing firms, ultimately keeping costs low.

The BMAP system utilizes a new concentric ring source-collector photodiode technology $(d=0.75,2.46 \mathrm{~mm})$ with a center-to-center spacing of $4.5 \mathrm{~mm}$; this reduces interpixel cross talk and creates photon traversal paths that interrogate just beyond $2 \mathrm{~mm}$ in depth depending on $\mu_{\mathrm{s}}^{\prime}$ and $\mu_{\mathrm{a}}$. A compressive sensing strategy is leveraged to maintain the intensity $[I(\lambda)]$ 
information content using a reduced set of eight discrete wavelengths. The sensing strategy is motivated by the need to capture the power spectral density in a serial fashion as the system does not utilize diffraction gratings or prisms; the number of wavelengths dictates the minimum acquisition time for a single spectral snapshot. The BMAP system uses a scalable Monte-Carlo model to invert measured reflectance into estimates of the absorption and reduced scattering coefficient $\left[\mu_{\mathrm{s}}^{\prime}(\lambda)\right.$ and $\left.\mu_{\mathrm{a}}(\lambda)\right]$. Such an inversion that is applied to remitted flux at a location near the point of entry is complicated by the influence of the scattering phase function, as described in Ref. 22. The authors are primarily concerned with differentiating cancerous regions against a reasonably contrasted, adipose background and, therefore, assume a homogeneous size distribution of scatterers as this has been shown to effectively distinguish tissue subtypes in corroboration with histopathological assessment. Stated clearly, the authors ignore the veracity of $\mu_{\mathrm{s}}^{\prime}(\lambda)$ in circumstances where the Henyey-Greenstein phase function does not necessarily apply.

One distinguishing clinical advantage of the BMAP system is afforded through the development of the CNC imaging platform, which provides a sixfold improvement in spatial resolution relative to the native PDA detector pitch $(0.75 \mathrm{~mm}$ compared to $4.5 \mathrm{~mm}$ ) and precision pressure control $[1-50 \pm 0.6 \mathrm{~mm} \mathrm{Hg}]$ at the probe-tissue interface during routine clinical use. The key motivators for developing the imaging platform were increasing the sensitivity to small, focal regions of disease on the margin through submillimeter sampling and providing robust quality control through repeatable contact pressure.

Routine clinical use consists of a sequential raster-scan during which the probe cyclically establishes contact with tissue, measures $I(\lambda)$, returns to a safe clearance height, and finally is translated in $x$ or $y$ according to the scan sequence. The cycle repeats until the entire margin is surveyed at the desired sampling resolution $(\Delta s)$ in $x$ and $y$. Within a clinical scan, each placement yields 16 simultaneous independent measures of $I(\lambda)$, resulting in 576 total spectra for each 36-placement raster-scan. Comprehensive surveillance of the margin landscape is thus achieved through 576 separate optical parameter estimates (via Monte-Carlo look-up table inversions) modulated in position. This parameter-dense map is interpolated using a bicubic spline to best represent the inherent optical property values: heterogeneities beneath the most superficial layer will contribute to the estimates and interpolation provides an effective average-like representation of the sensing volume, particularly with decreasing $\Delta s$. Stated differently, because information content is captured in depth and a tomographic reconstruction algorithm is not employed, the BMAP system can capture and accurately assess the disease status of features nearly equal to $\Delta s$ for $\Delta s \geq 0.75 \mathrm{~mm}$ using an interpolated 2-D representation of the margin in typical circumstances.

The current gold margin sampling standard is postoperative histopathology, wherein the excised tissue is fixed and sliced into 3 to $5 \mathrm{~mm}$ sections, and from these sections a 3 to $5 \mu \mathrm{m}$ shave is taken for microscopic review. At Duke University Medical Center, standard practice stipulates that a recommendation for re-excision surgery is only given if cancerous cells are found to be present at the margin edge, commonly referred to as "tumor on ink" and represents a positive margin status with all other circumstances constituting a negative margin. Historically, patients with cancer cells present within $2 \mathrm{~mm}$ of the margin edge were recommended for re-excision in the case of invasive disease; however, the movement toward all patients receiving postoperative radiation therapy has obviated the need for such re-excisions as it has been shown that the clinical outcome is the same. Still, postoperative radiation is a substantial clinical burden and may be unnecessary for disease greater than $2 \mathrm{~mm}$ from the margin edge, suggesting a critical circumstance in which the BMAP could alter the standard of care in providing such distance information. Furthermore, there are sincere sampling limitations of clinical pathology in that, due to the time required to review slides, it is only feasible to sample every $3 \mathrm{~mm}$ at best, suggesting that focal regions of disease smaller than $3 \mathrm{~mm}$ may be missed, putting the patient at risk for disease recurrence. Considering this, the BMAP device is uniquely positioned to reduce complications caused by missed disease as a morphological assessment is made every $0.75 \mathrm{~mm}$, far from the standard $3 \mathrm{~mm}$. The limitation of the BMAP in this regard is the lack of cellular resolution which may be necessary if the focal region of disease is a few cells in width; although unlikely, this may be caught by pathology but could not be correctly identified by the BMAP device due to its resolution limits. In terms of the FOV, the BMAP detector footprint is slightly smaller than the average en face margin surface area $\left(38 \mathrm{~cm}^{2}\right)$ to avoid imaging artifacts from nontissue regions. For larger specimens, the advantage of the BMAP design makes it simple to scale to larger detector arrays as it is merely a silicon chip; we have developed a prototype $8 \times 8$ array that covers four times the current detector area FOV $\left(10.24 \mathrm{~cm}^{2}\right)$. Clinically, it would be most pragmatic to have several probe sizes that can be selected based on the approximate specimen size available to the user, particularly considering the extremely low cost of mass produced silicon chips.

The control of pressure is imperative: user-associated error stemming from shaky, highly variable probe placement and pressure at the probe-tissue interface is avoided, preventing artifacts in extracted optical properties that may result in false-positive data due to tissue compression or insufficient contact. The elaborate effort to control and measure pressure at the probetissue interface stems from an evolving clinical standard-ofcare; radioactive localization seeds are now routinely used, and the ramifications of a misplaced seed require the tissue remained confined to a specialized container accessible only from the top side. Here, we demonstrate on gelatin-based multilayer solid tissue-simulating phantoms that the window in which optical parameter estimates remain valid is narrow, spanning 6 to $15 \mathrm{~mm} \mathrm{Hg}$. Although the photodiodes are sensitive at short integration times $(10 \mathrm{~ms})$, the time required to stabilize the applied pressure varies (typically from 50 to $250 \mathrm{~ms}$ ) for each placement during the raster-scanning procedure. One potential solution to this issue could involve removing contact pressure altogether; a contact-free embodiment utilizing small, cross-polarized relay lenslets could image the photodetector geometry directly onto the surface of the specimen. Note that the array design is quite amenable to this; the free-space illumination geometry allows for the polarization of the incoming light prior to passing through the illumination apertures. Likewise, the detectors could utilize a polarizing film oriented 90 from the illumination polarizer in lieu of the Teflon film, affording a cost-effective way to block any specular reflection. The feasibility of a cross-polarized noncontact point probe leveraging visible spectroscopy was demonstrated in Ref. 23 and achieved optical property 
Table 4 Optical imaging margin assessment tools.

\begin{tabular}{|c|c|c|c|c|c|c|}
\hline \multirow[b]{2}{*}{ Performance metrics ${ }^{\star}$} & \multicolumn{6}{|c|}{ System/reference } \\
\hline & $8 \mathrm{ch}^{9}$ & $49 \mathrm{ch}^{16}$ & BMAP & Ref. 24 & Refs. 25 and 28 & Ref. 30 \\
\hline Modality & DRS & DRS & CDRS & DRS/LIFS & SFDI & PSOCT \\
\hline Imaging $\tau$ & 5.97 & 0.27 & 0.31 & 0.05 & 0.02 & N/A \\
\hline Resolution (mm) & 5 & 0.75 & 0.75 & 0.25 & 1.25 & 0.012 \\
\hline Contrast & {$\left[\beta_{c}\right],\left\langle\mu_{\mathrm{s}}^{\prime}\right\rangle_{\lambda}$} & {$\left[\beta_{c}\right],\left\langle\mu_{\mathrm{s}}^{\prime}\right\rangle_{\lambda}$} & {$\left[\beta_{c}\right],\left\langle\mu_{\mathrm{s}}^{\prime}\right\rangle_{\lambda}$} & {$\left[\beta_{c}\right], \mathrm{NADH}$} & $\mu_{\mathrm{s}}^{\prime}, \mu_{\mathrm{a}}$ & $\mathrm{BR}, \mathrm{n}$ \\
\hline Size $\left(\mathrm{cm}^{3}\right)$ & $122 \times 76 \times 106$ & $60 \times 60 \times 27$ & $30 \times 25 \times 30$ & $60 \times 30 \times 30$ & $23 \times 19 \times 22$ & N/A \\
\hline Contact & $\mathrm{Y}, \mathrm{HH}$ & $\mathrm{Y}, \mathrm{HH}$ & $\mathrm{Y}$, Auto & $\mathrm{Y} / \mathrm{N}^{*}$ & $\mathrm{~N}$ & Y \\
\hline DX type & AA & AA & AA & $A A$ & AA & Path/R \\
\hline
\end{tabular}

*Note: Metrics are approximated from available literature.

extraction accuracy similar to that reported in this manuscript (\% error $\mu_{\mathrm{a}}=3.46 ; \mu_{\mathrm{s}}^{\prime}=8.42$ ).

There are several emerging technologies that share similarities with the BMAP system (see Appendix A.4, Table 4) and others that avoid some of the necessary innovations of this work, potentially at the expense of size, cost, and manufacturability. In the study outlined in Ref. 24, a scanning point probe was used to extract [ $\beta$-carotene] and collagen extent [via Nicotinamide adenine dinucleotide (NADH) fluorescence] as descriptor variables, as is the case with the BMAP system. One clinical advantage of the BMAP system over a single point-scanning technology is the native wide-field, multiplexed geometry; for similar integration times, the total scan time is reduced by a factor of 16.

Spatial frequency domain imaging (SFDI) is an existing wide-field technique that can obtain tissue optical properties by measuring the intensity of diffusely reflected light without making physical contact with the specimen. ${ }^{25-28}$ SFDI offers a distinct advantage in estimating tissue optical properties as it is possible to accurately decouple $\mu_{\mathrm{a}}(\lambda)$ and $\mu_{\mathrm{s}}^{\prime}(\lambda)$ from one another at different depths using multiple spatial frequencies and an analytical model for photon migration. Most SFDI-based approaches leverage a diffusion approximation to the radiative transport equation, as detailed in Ref. 29, resulting in fast, computationally efficient optical property estimates. The primary limitation of current SFDI systems relates back to clinical translatability; most utilize large, research-grade CCD cameras similar to our previous generation QDRI systems; however, transitioning to a commercially low-cost sensor equivalent would be nontrivial as high-resolution, sensitive cameras are needed to capture the high-frequency intensity variations specific to SFDI.

An Optical coherence tomography (OCT) needle-based technique described in Ref. 30 demonstrates an alternative approach with the added benefit of birefringence sensitivity, which is shown to be an effective dimension for distinguishing cancer from its dysplastic stroma, a current limitation of the BMAP system. The OCT needle exhibits high contrast across tissue subtypes but still requires trained personnel to score the images, constraining the utility to available clinical resources.

In conclusion, the authors believe that the BMAP system is a pragmatic solution to the margin assessment problem: the portability, accuracy, and manufacturability provide a realistically translatable path for integration into the clinical standard of care.

\section{Appendix: System Design}

\section{A.1 Light Source Design}

The light source consists of an Asahi Xenon arc lamp with an 8slot filter wheel housing 10-nm bandpass filters. A hybrid plastic LG serves as the conduit between the lamp and a custom absorbing tube. The absorbing tube is made of ABS plastic and is painted matte black on the inner walls to absorb any stray light and prevent high-angle, reflected light from entering the PDA apertures. Although the aperture throughput is roughly uniform, the free-space light delivery design comes at the expense of overall throughput and efficiency: the aperture throughput ranged from 17 to $22 \mu \mathrm{W}$ per aperture while the total output from the hybrid LG ranged from 45 to $55 \mathrm{~mW}$. Future iterations of the system can be replaced with energy efficient LEDs and guided light delivery. A custom dovetail style mount (also ABS plastic) is fastened to the topside of the printed circuit board and properly aligns the absorbing tube centrally to the surface normal of the $160.75-\mathrm{mm}$ etched apertures of the PDA [Fig. 10(a)]; the 4-post dovetail design ensures that the absorbing tube cannot be angled or off-center. The imaging probe is attached to the imaging platform via the absorbing tube; a small protrusion on the tube sidewall matched to the platform clamp ensures the probe is always aligned and level with the translational axes of the imaging platform. The power spectral density as measured at the end of the LG prior to free-space propagation is shown in Fig. 10(b). The maximum filter switching rate is $300 \mathrm{~ms}$ per filter and is the rate limiting process in data acquisition.

\section{A.2 Amplifier Design}

The integrating transimpedance amplifier system converts the raw photocurrent to easily measured voltage that can be read by a laptop computer. The basic system design is shown in Fig. 11(a). Note that direct photocurrent measurements are 
(a)
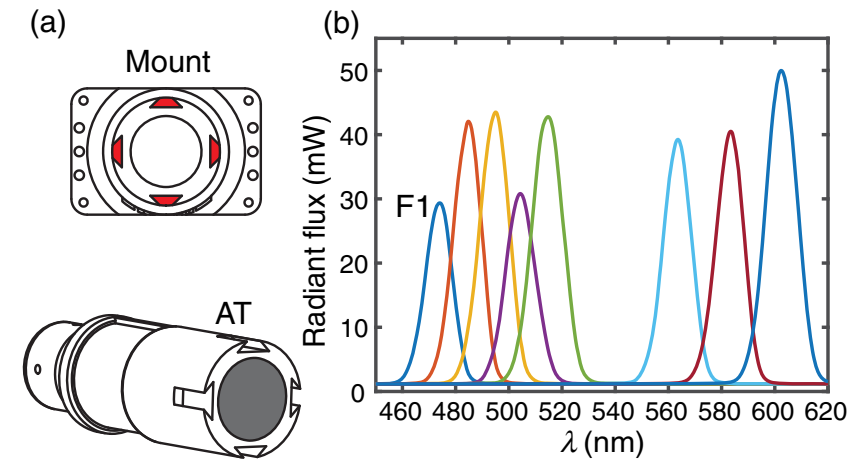

Fig. 10 Light source design. In (a), the absorbing tube and the corresponding $A B S$ plastic mount with four dove-tail protrusions that ensure proper alignment of the tube and PDA. (b) The radiant flux as measured at the LG output.

not feasible nor cost-effective due to the necessary ammeter sensitivity and dynamic range required to measure electric currents spanning $10^{-6}$ to $10^{-12} \mathrm{~A}$. The amplifier design consists of a precision (16-bit) USB DAQ (NI-USB-6212, National Instruments, Austin, TX) providing 16 separate analog voltage inputs, a custom-made 4-layer ITIA board (printed by Advanced Circuits, LLC) with separate analog and digital ground planes to reduce EMI, an Arduino-based embedded control unit providing the TTL timing signals based on the required integration time, a standalone low-noise power supply with isolated $+15 \mathrm{~V}$, $-15 \mathrm{~V}$, and $+5 \mathrm{~V}$ rails, and finally a USB hub connecting the Arduino, DAQ, and panel mount connectors to receive USB signals from external components. A single USB (type B) panel mount connector is used to link the hub to the laptop computer. Custom firmware for the ITIA system leverages a modified version of the open-source LabVIEW ${ }^{\text {TM }}$ interface for Arduino and allows a wide range of integration times (500 $\mu$ s to $>100 \mathrm{~s})$.

Due to the difficulty in obtaining silicon wafers for university volume use, the amplifier was designed to support and interface both pnp and npn style PDAs. There are two inverting op-amp stages downstream each of the 16 ITIA ICs on the amplifier board. The first op-amp stage scales the 0 to $+13 \mathrm{~V}$ output of ITIA IC to span 0 to $-5 \mathrm{~V}$, and the second inverts this output. Depending on the type of PDA used, a set of jumpers can be switched to ensure the output is always 0 to $5 \mathrm{~V}$ [Fig. 11(b)].

\section{A.3 Imaging Platform Design}

The imaging platform and an exploded view of key components are diagrammed in Fig. 12(a), with the interchangeable arms and force sensors highlighted in red. The interchangeable arms consist of a two-piece motif wherein an accessory is fastened to a fixed length dovetail spacing arm. The goal in using this design is so the components can be frequently removed and replaced rapidly without tools. As seen in Fig. 12(b), the two dovetail protrusions at the distal end are arranged perpendicularly; this arrangement ensures that there is no wiggle of the arm in the $x y z$ directions while the entire module can easily be inserted or removed by the user. Furthermore, the fixed length interchangeable segment provides a way to change the geometry and extent of reach of attachments without requiring changes to the clamping portion. The importance of this is made obvious in the consideration of a newer surgical protocol involving radioactive seeds; instead of using a placement wire, some surgeons elect to insert a radioactive seed in the tumor to help guide where the center of mass is, and it is imperative that the seed is not lost during the procedure. Thus, the lumpectomy cannot be removed from a specialized (opaque) container in which it is placed immediately after it leaves the patient, meaning that the optical assessment must be performed while the specimen remains inside this container. To achieve adequate probe contact with the specimen while inside this container, a specialized angled arm is required; in cases where this is needed, the user can swap the straight arm for the angled one without removing the probe or camera from its respective clamp. Each clamp is designed to share an optical axis; this reduces the number of components necessary to adapt to various clinical scenarios as the clamp can be attached to any style arm and is guaranteed to share an optical axis with other accessories. In Fig. 12(c), a digital image of the platform is shown with the USB camera mounted in lieu of the PDA. To reduce clinical clutter, the (a)

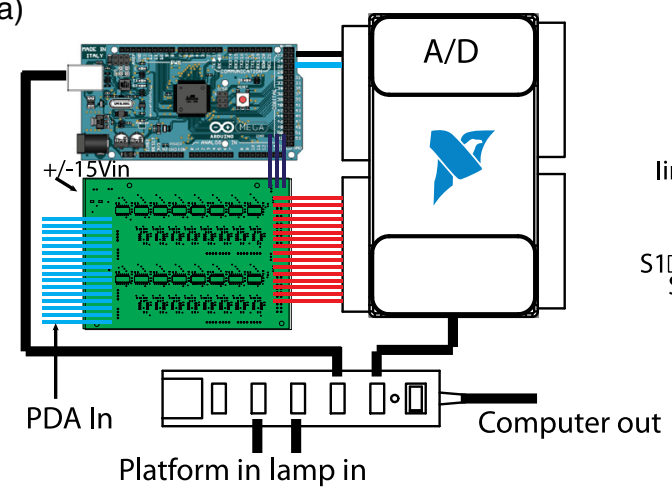

(b)

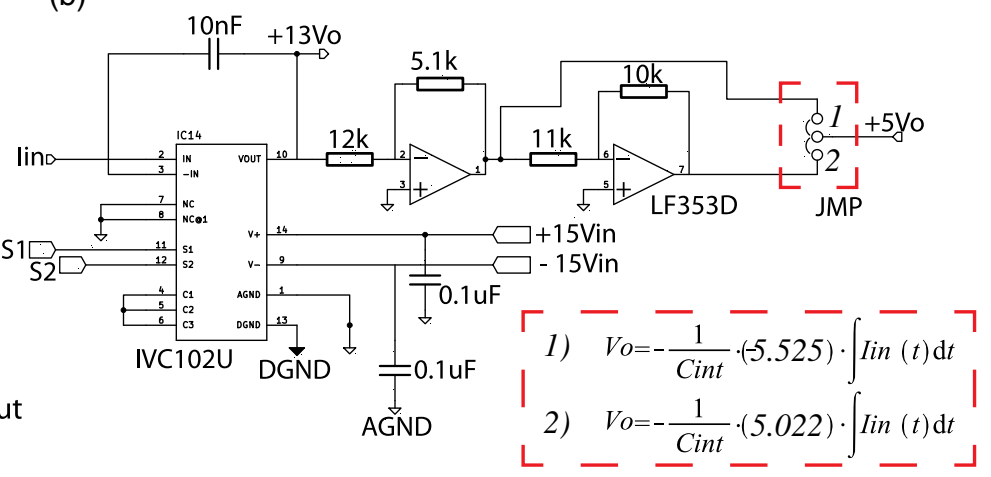

Fig. 11 Amplifier design: (a) the amplifier design, consists of an Arduino microcontroller to clock the charge capture and release of integrating amplifier ICs, an NI USB analog-to-digital converter to capture the voltage readout from the ITIAs, and a USB hub to end the daisy chain of instruments and provide a single USB output for communication with a laptop. (b) The schematic of the ITIAs demonstrating the suitability for both pnp and npn PDAs with dual-stage inverting op-amps. The gain of each stage is shown in the dashed red box. 

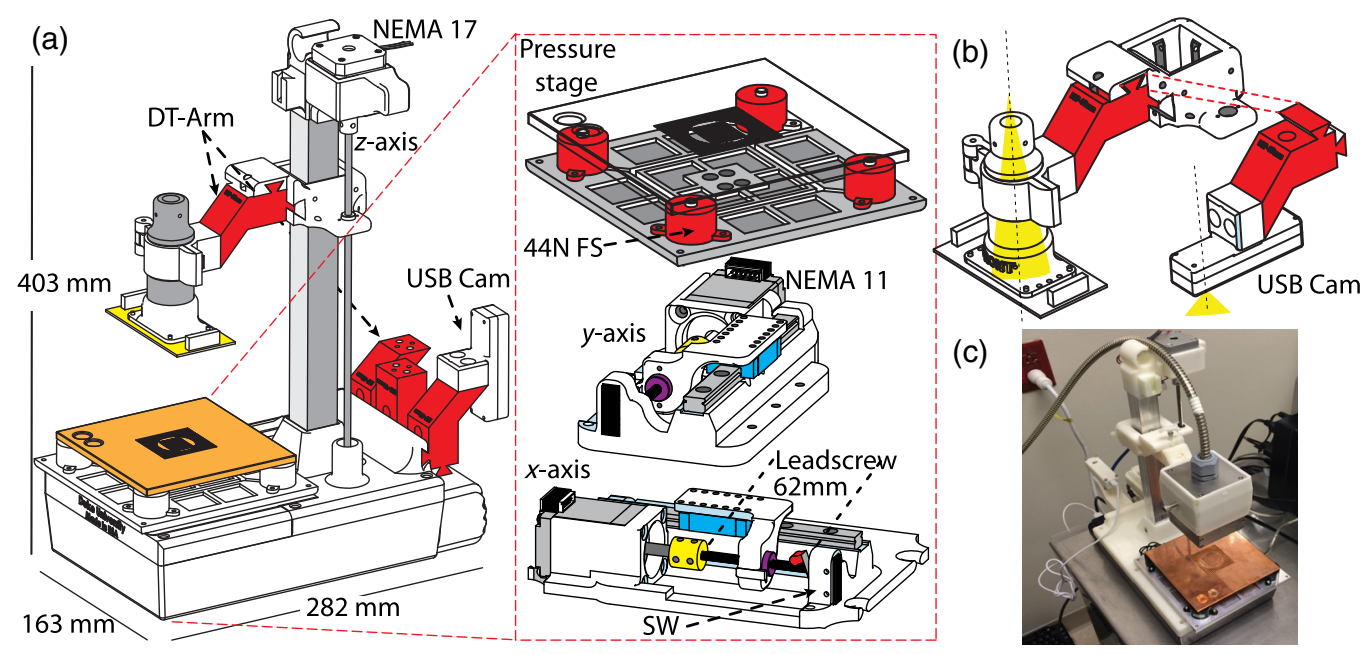

Fig. 12 Imaging platform design: (a) diagram of full imaging platform with close-up of pressure sensing base, $x$-translational axis, and $y$-translational axis, (b) close-up of interchangeable arm illustrating dovetail slot mechanism that allows the user to swap components without tools, and (c) a digital image with the imaging platform just prior to clinical use.

stock of arms and accessories is conveniently mounted to a storage rail located on the backside of the imaging platform.

Each of the translational axes is a custom-made leadscrewbased linear actuator designed to provide fine incremental motion yet move rapidly as to not significantly slow the raster-scanning acquisition process. The lateral translational axes $(x, y)$ are connected to the pressure sensing stage and utilize a fine leadscrew (1:0.609 rev:mm travel, $l=62 \mathrm{~mm})$ (McMaster-Carr, Inc., Douglasville, Georgia, \#6642k21) fixed to dual-phase NEMA 11 stepper motors with 1.8 deg angular resolution, providing the ability to move in increments as small as $3.3 \mu \mathrm{m}$. These are used with wear-compensating nuts to reduce backlash resulting from extended use. Positional uncertainty (backlash) is $\leq 10 \mu \mathrm{m}$ upon direction reversal. The $x, y$ actuators are diagrammed in Fig. 12(a). The vertical axis $(z)$ utilizes a multistart, fast-travel leadscrew with a travel distance ratio of 1:9.407 (rev:mm) to move the probe and apply pressure quickly. The $z$-axis has over $200 \mathrm{~mm}$ of travel to compensate for the previously mentioned specimen containers and the respective angled arms. A NEMA 17 stepper motor ( $\Delta \angle=1.8 \mathrm{deg}$ ) is used for increased holding torque as the rail is load bearing and the probe must be locked into place once the target pressure is reached. The smallest $\Delta z$ for the $z$-axis is $47 \mu \mathrm{m}$; this corresponds to a pressure resolution $\Delta P=$ $0.6 \mathrm{~mm} \mathrm{Hg}$ for the typical clinical specimen.

The stepper motor system is powered by a custom-made 4-channel H-bridge driver designed to output a constant current such that the voltage may fluctuate as the speed of the motor is changed; the constant current output obviates the need to add logic for acceleration/deceleration and provides the fastest mode of translation. Each axis is equipped with a bumper-switch to indicate the end of travel and provides a calibration reference position such that the platform can move to a home position on startup. The platform utilizes an Arduino-based embedded system control similar to the transimpedance amplifier, also leveraging a modified version of the open-source LabVIEW ${ }^{\text {TM }}$ interface for Arduino. The mode of operation is designed to be similar to most CNC machining tools; G-code-like commands are sent to the Arduino via LabVIEWTM. The pressure feedback loop and absolute $x y z$ position are managed entirely on the embedded system; these functions must be real time to avoid overcompression of the specimen and/or loss of position coordinates. The user interface periodically $(150 \mathrm{~ms})$ polls the embedded system to retrieve current values for position and pressure.

\section{A.4 Imaging Modality Comparisons}

Definitions for table: Imaging $\tau$ represents a characteristic time constant incorporating the resolution $(\Delta s)$ and the time required to capture an image over the given FOV, as described below

$\tau=\frac{\Delta s^{2} \cdot t(s)}{\mathrm{FOV}}$

where $\Delta s$ is the sampling resolution in $\mathrm{mm}, t(s)$ is the time in seconds required for a single full-spectrum snapshot, and FOV is the field of view in $\mathrm{cm}^{2}$. Note that larger values suggest lower resolution, smaller FOV, and longer acquisition times. Resolution values are nominal for clinical use. DRS, diffuse reflectance spectroscopy; CDRS, compressive DRS; LIFS, laser-induced fluorescence spectroscopy; SFDI, spatial frequency domain imaging; PSOCT, polarization sensitive optical coherence tomography; $\mathrm{HH}$, hand-held device; AA, automated algorithm; path/R, pathologist/trained reader; BR, birefringence, and $n$, index of refraction.

\section{Disclosures}

Dr. Ramanujam owns stock in and has been employed by or provided consulting services for Zenalux Biomedical, Inc., a company that developed portions of the 49ch technology described in this paper. Zenalux Biomedical has licenses or options to license intellectual property invented by Dr. Ramanujam. Zenalux Biomedical did not support any of the original work reported in this paper, either in cash or in kind.

\section{Acknowledgments}

This project was supported by the National Institutes of Health (NIH) Grant No. R01EB011574-01A1. The NIH provided 
support in the form of salaries for authors A.L., G.P., O.S., D.M., M.B., N.J., and N.R. but did not have any additional role in the study design, data collection and analysis, decision to publish, or preparation of the paper. The National Science Foundation graduate research fellowship program provided support in the form of salary for author B.N. The 49ch device was developed under support by the Department of Defense Grant No. W81XWH-09-1-0410 and by the Small Business Technology Transfer Phase II Grant No. 2R42CA128160-02 Fast Spectral Imaging Device for Tumor Margin Mapping awarded to Zenalux, Inc. and Duke University.

\section{References}

1. J. Ferlay et al., "Cancer incidence and mortality worldwide: sources, methods and major patterns in GLOBOCAN 2012," Int. J. Cancer 136(5), E359-E386 (2015).

2. D. Lazovich et al., "Underutilization of breast-conserving surgery and radiation therapy among women with stage I or II breast cancer," J. Am. Med. Assoc. 266(24), 3433-3438 (1991).

3. C. Sanchez et al., "Factors associated with re-excision in patients with early-stage breast cancer treated with breast conservation therapy," Am. Surg. 76(3), 331-334 (2010).

4. L. G. Wilke et al., "Repeat surgery after breast conservation for the treatment of stage 0 to II breast carcinoma: a report from the National Cancer Data Base, 2004-2010," JAMA Surg. 149(12), 1296-1305 (2014).

5. E. R. Camp et al., "Minimizing local recurrence after breast conserving therapy using intraoperative shaved margins to determine pathologic tumor clearance," J. Am. Coll. Surg. 201(6), 855-861 (2005).

6. T. P. Olson et al., "Frozen section analysis for intraoperative margin assessment during breast-conserving surgery results in low rates of re-excision and local recurrence," Ann. Surg. Oncol. 14(10), 29532960 (2007).

7. G. M. Palmer and N. Ramanujam, "Monte Carlo-based inverse model for calculating tissue optical properties. Part I: theory and validation on synthetic phantoms," Appl. Opt. 45, 1062-1071 (2006).

8. J. Q. Brown et al., "Optical spectral surveillance of breast tissue landscapes for detection of residual disease in breast tumor margins," PLoS One 8(7), 8-12 (2013).

9. T. M. Bydlon et al., "Optical spectral imaging for breast margin assessment: a comprehensive assessment of sources of contrast," in Biomedical Optics and 3-D Imaging, BW2B.4 (2012).

10. H. L. Fu et al., "A low-cost, portable, and quantitative spectral imaging system for application to biological tissues," Opt. Express 18(12), 12630-12645 (2010).

11. J. Y. Lo et al., "Wavelength optimization for quantitative spectral imaging of breast tumor margins," PLoS One 8(4), e61767 (2013).

12. S. Dhar et al., "A diffuse reflectance spectral imaging system for tumor margin assessment using custom annular PDAs," Biomed. Opt. Express 3, 3211 (2012).
13. C. Zhu et al., "Diagnosis of breast cancer using diffuse reflectance spectroscopy: comparison of a Monte Carlo versus partial least squares analysis based feature extraction technique," Lasers Surg. Med. 38(7), 714-724 (2006).

14. J. E. Bender et al., "A robust Monte Carlo model for the extraction of biological absorption and scattering in vivo," IEEE Trans. Biomed. Eng. 56(4), 960-968 (2009).

15. R. Akhtar et al., "Characterizing the elastic properties of tissues," Mater. Today 14(3), 96-105 (2011).

16. B. S. Nichols et al., "A quantitative diffuse reflectance imaging (QDRI) system for comprehensive surveillance of the morphological landscape in breast tumor margins," PLoS One 10(6), e0127525 (2015).

17. K. Zhang et al., "The collagen receptor discoidin domain receptor 2 stabilizes SNAIL1 to facilitate breast cancer metastasis," Nat. Cell Biol. 15(6), 677-687 (2013).

18. K. Riching et al., "3D collagen alignment limits protrusions to enhance breast cancer cell persistence," Biophys. J. 107(11), 2546-2558 (2014).

19. P. P. Provenzano and P. J. Keely, "The role of focal adhesion kinase in tumor initiation and progression," Cell Adhes. Migr. 3(4), 347-350 (2009).

20. C. Shannon and I. E. Smith, "Breast cancer in adolescents and young women," Eur. J. Cancer 39(18), 2632-2642 (2003).

21. N. F. Boyd et al., "Breast tissue composition and susceptibility to breast cancer," J. Natl. Cancer Inst. 102(16), 1224-1237 (2010).

22. E. Vitkin et al., "Photon diffusion near the point-of-entry in anisotropically scattering turbid media," Nat. Commun. 2, 587 (2011).

23. S. F. Bish et al., "Development of a noncontact diffuse optical spectroscopy probe for measuring tissue optical properties," J. Biomed. Opt. 16(12), 120505 (2011).

24. N. Lue et al., "Portable optical fiber probe-based spectroscopic scanner for rapid cancer diagnosis: a new tool for intraoperative margin assessment," PLoS One 7(1), e30887 (2012).

25. A. M. Laughney et al., "System analysis of spatial frequency domain imaging for quantitative mapping of surgically resected breast tissues," J. Biomed. Opt. 18(3), 036012 (2013).

26. S. C. Kanick et al., "Sub-diffusive scattering parameter maps recovered using wide-field high-frequency structured light imaging," Biomed. Opt. Express 5(10), 3376-3390 (2014).

27. B. J. Tromberg et al., "Non-invasive measurements of breast tissue optical properties using frequency-domain photon migration," Philos. Trans. R. Soc. B 352(1354), 661-668 (1997).

28. A. Cerussi et al., "In vivo absorption, scattering, and physiologic properties of 58 malignant breast tumors determined by broadband diffuse optical spectroscopy," J. Biomed. Opt. 11(4), 044005 (2006).

29. V. Venugopalan, J. You, and B. Tromberg, "Radiative transport in the diffusion approximation: an extension for highly absorbing media and small source-detector separations," Phys. Rev. E 58(2), 2395-2407 (1998).

30. M. Villiger et al., "Deep tissue volume imaging of birefringence through fibre-optic needle probes for the delineation of breast tumour," Sci. Rep. 6(February), 28771 (2016).

Biographies for the authors are not available. 